\title{
Calculus for Directional Limiting Normal Cones and Subdifferentials
}

\author{
Matúš Benko ${ }^{1}$ (D) . Helmut Gfrerer ${ }^{1}$ - Jiří V. Outrata ${ }^{2,3}$
}

Received: 12 December 2017 / Accepted: 3 August 2018 / Published online: 14 August 2018

(C) The Author(s) 2018

\begin{abstract}
The paper is devoted to the development of a comprehensive calculus for directional limiting normal cones, subdifferentials and coderivatives in finite dimensions. This calculus encompasses the whole range of the standard generalized differential calculus for (non-directional) limiting notions and relies on very weak (non-restrictive) qualification conditions having also a directional character. The derived rules facilitate the application of tools exploiting the directional limiting notions to difficult problems of variational analysis including, for instance, various stability and sensitivity issues. This is illustrated by some selected applications in the last part of the paper.
\end{abstract}

Keywords Generalized differential calculus · Directional limiting normal cone · Directional limiting subdifferential · Qualification conditions

Mathematics Subject Classification (2010) 49J53 · 49J52 · 90C31

\section{Introduction}

Since the early works of Mordukhovich, the limiting normal cone and the corresponding subdifferential belong to the central notions of variational analysis. They admit a rich calculus both in finite as well as in infinite dimensions and have been successfully utilized in

Matúš Benko

benko@numa.uni-linz.ac.at

Helmut Gfrerer

helmut.gfrerer@jku.at

Jiří V. Outrata

outrata@utia.cas.cz

1 Institute of Computational Mathematics, Johannes Kepler University Linz, A-4040 Linz, Austria

2 Czech Academy of Sciences, Institute of Information Theory and Automation, 18208 Prague, Czech Republic

3 Centre for Informatics and Applied Optimization, Federation University of Australia, Ballarat, Vic 3350, Australia 
a large variety of optimization and equilibrium problems, see [20, 22] and the references therein. In particular, these notions play an important role in stability and sensitivity issues, above all in analysis of various Lipschitzian stability notions related to multifunctions. Having been motivated by some of the above listed applications, in [14] the authors refined the original definitions by restricting the limiting process only to a subset of sequences used in the original definitions. This lead eventually to the notions of directional limiting normal cone and directional limiting subdifferential which have been further developed and utilized above all in the works authored or coauthored by Gfrerer [6-13]. It turned out that these directional notions (together with the directional limiting coderivative) enable us indeed a substantially finer analysis of situations in which the estimates, provided by the standard calculus, are too rough and so the corresponding assertions are not very useful. This is, e.g., the case of verification of metric subregularity of feasibility mappings (calmness of perturbation mappings) related to constraint systems, which lead to new first- and second-order sufficient conditions for metric subregularity $[6,7]$. They are now widely used as weak (nonrestrictive) but yet verifiable qualification conditions [10, 13, 24]. In [11] the authors used the directional limiting coderivative to establish new weak conditions ensuring the calmness and the Aubin property of rather general implicitly defined multifunctions and in [12] this technique has then been worked out for a class of parameterized variational systems.

Further, directional limiting coderivative appears in sharp first-order optimality conditions [6], entitled extended M-stationarity in [8], which provide a dual characterization of B-stationarity for disjunctive programs.

One can definitely imagine also numerous other problems of variational analysis in which the directional notions could be successfully employed. In all of them, however, one needs a set of rules enabling us to compute efficiently the directional normal cones and subdifferentials of concrete sets and functions in a similar way like in the standard generalized differential calculus of Mordukhovich. Some parts of such a calculus have already been conducted in connection with various results mentioned above. In particular, in [7] one finds, apart from some elementary rules, formulas for directional limiting normal cones to unions of convex polyhedral sets and in $[11,12]$ second-order chain rules have been derived which enable us to compute directional limiting coderivatives to normal-cone mappings associated with various types of sets. Further, [19] contains several rules of the directional calculus even in Banach spaces, in [23] the authors proved a special coderivative sum rule and in [24] the situation has been examined when one has to do with the Cartesian products of sets and mappings.

The aim of this paper is to fill in this gap by building a systematic decent calculus of directional limiting normal cones and subdifferentials following essentially the lines of [20, Chapter 3] and [16]. The structure is as follows.

In Section 2 we collect the needed definitions and present some auxiliary results used throughout the paper. Section 3 is devoted to the calculus of directional limiting normal cones. As the most important results we consider formulas for the directional limiting normal cone of the pre-image and of the image of a set in a Lipschitz continuous mapping. These results have numerous important consequences. Section 4 concerns the calculus of directional limiting subdifferentials. Apart from the chain and sum rules we consider the case of value functions, distance functions, pointwise minima and maxima and examine also the partial directional limiting subdifferentials. Section 5 provides formulas for directional limiting coderivatives of compositions and sums of multifunctions together with some important special cases. In Section 6 we present some problems of variational analysis, where the usage of the directional limiting calculus leads to weaker (less restrictive) sufficient conditions or sharper (more precise) estimates. 
Similarly as in [16], we have attempted to impose the "weakest" qualification conditions expressed mostly in terms of directional metric subregularity of associated feasibility mappings. Admittedly, these conditions are not always verifiable, but they can in many cases be replaced by stronger (more restrictive) conditions expressed in terms of problem data.

In the directional calculus one also meets a new specific difficulty associated with the computation of the images or the pre-images of the given direction in the considered mappings. This obstacle leads in some cases to more complicated rules or to additional qualification conditions.

It turns out, that the essential role in the calculus is played by Theorems 3.1 and 3.2, concerning the pre-image and the image of a set in a continuous/Lipschitz mapping. The basic ideas arising in these two statements appear in fact in almost all calculus rules throughout the whole paper.

Apart from the directional notions investigated in this paper there are several other constructions, where directions play an important role. Herewith we mean above all the quasidifferentials and exhausters of Demyanov [4], directed subdifferentials of Baier [1,2] and lexicographic derivatives of Nesterov [21]. In all of them, however, the directions are used as a vehicle to describe the local behavior of the considered sets or mappings and they do not pretend to capture the "directional" behavior.

The following notation is employed. The closed unit ball and the unit sphere in $\mathbb{R}^{n}$ are denoted by $\mathbb{B}$ and $\mathbb{S}$, respectively, while $\mathbb{B}_{r}(\bar{x}):=\left\{x \in \mathbb{R}^{n} \mid\|x-\bar{x}\| \leq r\right\}$. The identity mapping is denoted by Id. Given a set $\Omega \subset \mathbb{R}^{n}$, bd $\Omega$ stands for the boundary of $\Omega$, i.e., the set of points whose every neighborhood contains a point of $\Omega$ as well as a point not belonging to $\Omega$. Moreover, given also a point $\bar{x}, \mathrm{~d}_{\Omega}(\bar{x})$ denotes the distance from $\bar{x}$ to set $\Omega$ and $\mathrm{P}_{\Omega}(\bar{x})$ denotes the projection of $\bar{x}$ onto $\Omega$. For a sequence $x_{k}, x_{k} \stackrel{\Omega}{\rightarrow} \bar{x}$ stands for $x_{k} \rightarrow \bar{x}$ with $x_{k} \in \Omega$, while $x_{k} \stackrel{f}{\rightarrow} \bar{x}$ with $f: \mathbb{R}^{n} \rightarrow \overline{\mathbb{R}}$ being an extended real-valued function stands for $x_{k} \rightarrow \bar{x}$ with $f\left(x_{k}\right) \rightarrow f(\bar{x})$. Given a directionally differentiable function $\varphi: \mathbb{R}^{n} \rightarrow \mathbb{R}^{m}$ at $\bar{x} \in \operatorname{dom} \varphi, \varphi^{\prime}(\bar{x} ; h)$ denotes the directional derivative of $\varphi$ at $\bar{x}$ in direction $h$. Finally, following traditional patterns, we denote by $o(t)$ for $t \geq 0$ a term with the property that $o(t) / t \rightarrow 0$ when $t \downarrow 0$.

\section{Preliminaries}

We start by recalling several definitions and results from variational analysis. Let $\Omega \subset \mathbb{R}^{n}$ be an arbitrary closed set and $\bar{x} \in \Omega$. The contingent (also called Bouligand or tangent) cone to $\Omega$ at $\bar{x}$, denoted by $T_{\Omega}(\bar{x})$, is given by

$$
T_{\Omega}(\bar{x}):=\left\{u \in \mathbb{R}^{n} \mid \exists\left(u_{k}\right) \rightarrow u,\left(t_{k}\right) \downarrow 0: \bar{x}+t_{k} u_{k} \in \Omega \forall k\right\} .
$$

We denote by

$$
\hat{N}_{\Omega}(\bar{x}):=\left\{\xi \in \mathbb{R}^{n} \mid \limsup _{x^{\prime} \stackrel{\Omega}{\rightarrow} \bar{x}} \frac{\xi^{T}\left(x^{\prime}-\bar{x}\right)}{\left\|x^{\prime}-\bar{x}\right\|} \leq 0\right\}
$$

the Fréchet (regular) normal cone to $\Omega$ at $\bar{x}$. Finally, the limiting (Mordukhovich) normal cone to $\Omega$ at $\bar{x}$ is defined by

$$
N_{\Omega}(\bar{x}):=\left\{\xi \in \mathbb{R}^{n} \mid \exists\left(x_{k}\right) \stackrel{\Omega}{\rightarrow} \bar{x},\left(\xi_{k}\right) \rightarrow \xi: \xi_{k} \in \hat{N}_{\Omega}\left(x_{k}\right) \forall k\right\} .
$$

If $\bar{x} \notin \Omega$, we put $T_{\Omega}(\bar{x})=\emptyset, \hat{N}_{\Omega}(\bar{x})=\emptyset$ and $N_{\Omega}(\bar{x})=\emptyset$. 
The Mordukhovich normal cone is generally nonconvex whereas the Fréchet normal cone is always convex. In the case of a convex set $\Omega$, both the Fréchet normal cone and the Mordukhovich normal cone coincide with the standard normal cone from convex analysis and, moreover, the contingent cone is equal to the tangent cone in the sense of convex analysis.

Note that $\xi \in \hat{N}_{\Omega}(\bar{x}) \Leftrightarrow \xi^{T} u \leq 0 \forall u \in T_{\Omega}(\bar{x})$, i.e., $\hat{N}_{\Omega}(\bar{x})=\hat{N}_{T_{\Omega}(\bar{x})}(0)=T_{\Omega}(\bar{x})^{\circ}$ is the polar cone of $T_{\Omega}(\bar{x})$.

Consider an extended real-valued function $f: \mathbb{R}^{n} \rightarrow \overline{\mathbb{R}}$ and a point $\bar{x} \in \operatorname{dom} f:=$ $\left\{x \in \mathbb{R}^{n} \mid f(x) \in \mathbb{R}\right\}$, where $\operatorname{dom} f$ denotes the domain of $f$. The Fréchet (regular) subdifferential of $f$ at $\bar{x}$ is a set $\hat{\partial} f(\bar{x})$ consisting of all $\xi \in \mathbb{R}^{n}$ such that

$$
f(x) \geq f(\bar{x})+\langle\xi, x-\bar{x}\rangle+o(\|x-\bar{x}\|),
$$

the limiting (Mordukhovich) subdifferential of $f$ at $\bar{x}$, denoted by $\partial f(\bar{x})$, is given by

$$
\partial f(\bar{x}):=\left\{\xi \in \mathbb{R}^{n} \mid \exists\left(x_{k}\right) \stackrel{f}{\rightarrow} \bar{x},\left(\xi_{k}\right) \rightarrow \xi: \xi_{k} \in \hat{\partial} f\left(x_{k}\right) \forall k\right\}
$$

and the singular subdifferential of $f$ at $\bar{x}$ is defined by

$$
\partial^{\infty} f(\bar{x}):=\left\{\xi \in \mathbb{R}^{n} \mid \exists\left(\lambda_{k}\right) \downarrow 0,\left(x_{k}\right) \stackrel{f}{\rightarrow} \bar{x}, \xi_{k} \in \hat{\partial} f\left(x_{k}\right) \forall k:\left(\lambda_{k} \xi_{k}\right) \rightarrow \xi\right\} .
$$

Denoting by epi $f:=\left\{(x, \alpha) \in R^{n+1} \mid a \geq f(x)\right\}$ the epigraph of $f$, there is a wellknown equivalent description of the subdifferentials, namely

$$
\begin{aligned}
& \hat{\partial} f(\bar{x})=\left\{\xi \in \mathbb{R}^{n} \mid(\xi,-1) \in \hat{N}_{\text {epi } f}(\bar{x}, f(\bar{x}))\right\}, \\
& \partial f(\bar{x})=\left\{\xi \in \mathbb{R}^{n} \mid(\xi,-1) \in N_{\text {epi } f}(\bar{x}, f(\bar{x}))\right\}, \\
& \partial^{\infty} f(\bar{x})=\left\{\xi \in \mathbb{R}^{n} \mid(\xi, 0) \in N_{\text {epi } f}(\bar{x}, f(\bar{x}))\right\},
\end{aligned}
$$

where the last relation holds if epi $f$ is locally closed around $(\bar{x}, f(\bar{x}))$ or, equivalently, $f$ is lower semicontinuous (lsc) around $\bar{x}$, see e.g. [22, Theorem 8.9].

Given a multifunction $M: \mathbb{R}^{n} \rightrightarrows \mathbb{R}^{m}$ and a point $(\bar{x}, \bar{y}) \in \operatorname{gph} M:=\left\{(x, y) \in \mathbb{R}^{n} \times\right.$ $\left.\mathbb{R}^{m} \mid y \in M(x)\right\}$, where gph $M$ denotes the graph of $M$, the graphical derivative of $M$ at $(\bar{x}, \bar{y})$ is a multifunction $D M(\bar{x}, \bar{y}): \mathbb{R}^{n} \rightrightarrows \mathbb{R}^{m}$ given by

$$
D M(\bar{x}, \bar{y})(u):=\left\{v \in \mathbb{R}^{m} \mid(u, v) \in T_{\operatorname{gph} M}(\bar{x}, \bar{y})\right\} .
$$

The regular coderivative of $M$ at $(\bar{x}, \bar{y})$ is a multifunction $\hat{D}^{*} M(\bar{x}, \bar{y}): \mathbb{R}^{m} \rightrightarrows \mathbb{R}^{n}$ with the values

$$
\hat{D}^{*} M(\bar{x}, \bar{y})(\eta):=\left\{\xi \in \mathbb{R}^{n} \mid(\xi,-\eta) \in \hat{N}_{\mathrm{gph} M}(\bar{x}, \bar{y})\right\}
$$

and the limiting (Mordukhovich) coderivative of $M$ at $(\bar{x}, \bar{y})$ is a multifunction $D^{*} M(\bar{x}, \bar{y})$ : $\mathbb{R}^{m} \rightrightarrows \mathbb{R}^{n}$ defined by

$$
D^{*} M(\bar{x}, \bar{y})(\eta):=\left\{\xi \in \mathbb{R}^{n} \mid(\xi,-\eta) \in N_{\mathrm{gph} M}(\bar{x}, \bar{y})\right\},
$$

i.e., $D^{*} M(\bar{x}, \bar{y})(\eta)$ is the collection of all $\xi \in \mathbb{R}^{n}$ for which there are sequences $\left(x_{k}, y_{k}\right) \rightarrow$ $(\bar{x}, \bar{y})$ and $\left(\xi_{k}, \eta_{k}\right) \rightarrow(\xi, \eta)$ with $\left(\xi_{k},-\eta_{k}\right) \in \hat{N}_{\mathrm{gph} M}\left(x_{k}, y_{k}\right)$, or, equivalently, $\xi_{k} \in$ $\hat{D}^{*} M\left(x_{k}, y_{k}\right)\left(\eta_{k}\right)$. The notation $D M(\bar{x}, \bar{y}), \hat{D}^{*} M(\bar{x}, \bar{y})$ and $D^{*} M(\bar{x}, \bar{y})$ is simplified to $D M(\bar{x}), \hat{D}^{*} M(\bar{x})$ and $D^{*} M(\bar{x})$ when $M$ is single-valued at $\bar{x}$ with $M(\bar{x})=\{\bar{y}\}$.

Just as in case of subdiferentials and epigraphs, it is often important to have closed graphs of multifunctions. We say that $M$ is outer semicontinuous (osc) at $\bar{x}$ if the existence of sequences $x_{k} \rightarrow \bar{x}$ and $y_{k} \rightarrow y$ with $y_{k} \in M\left(x_{k}\right)$ implies $y \in M(\bar{x})$ and we say that $M$ is osc if it is osc at every point, which is equivalent to the closedness of gph $M$, see [22, Theorem 5.7].

For more details we refer to the monographs [20, 22]. 
Directional versions of these limiting constructions were introduced in [14] and [6] for general Banach spaces and later on equivalently reformulated for finite dimensional spaces in [8] in the following way. Given a direction $u \in \mathbb{R}^{n}$, the limiting normal cone to a subset $\Omega \subset \mathbb{R}^{n}$ at $\bar{x} \in \Omega$ in direction $u$ is defined by

$$
N_{\Omega}(\bar{x} ; u):=\left\{\xi \in \mathbb{R}^{n} \mid \exists\left(t_{k}\right) \downarrow 0,\left(u_{k}\right) \rightarrow u,\left(\xi_{k}\right) \rightarrow \xi: \xi_{k} \in \hat{N}_{\Omega}\left(\bar{x}+t_{k} u_{k}\right) \forall k\right\} .
$$

Note that by the definition we have $N_{\Omega}(\bar{x} ; 0)=N_{\Omega}(\bar{x})$. Further $N_{\Omega}(\bar{x} ; u) \subset N_{\Omega}(\bar{x})$ for all $u, N_{\Omega}(\bar{x} ; u)=\emptyset$ if $u \notin T_{\Omega}(\bar{x})$ and $N_{\Omega}(\bar{x} ; u)=\{0\}$ if $u \in T_{\Omega}(\bar{x}) \backslash T_{\mathrm{bd} \Omega}(\bar{x})$.

The following simple lemma provides a hint about possible applications of directional limiting normal cones.

Lemma 2.1 Let $\Omega \subset \mathbb{R}^{n}$ be closed and $\bar{x} \in \Omega$. Then

$$
N_{\Omega}(\bar{x})=\hat{N}_{\Omega}(\bar{x}) \cup \bigcup_{u \in T_{\Omega}(\bar{x}) \cap \mathbb{S}} N_{\Omega}(\bar{x}, u) .
$$

Proof Inclusion $\supset$ follows directly from definition.

Now let $\xi \in N_{\Omega}(\bar{x})$ and consider $x_{k} \rightarrow \bar{x}, \xi_{k} \rightarrow \xi$ with $x_{k} \in \Omega$ and $\xi_{k} \in \hat{N}_{\Omega}\left(x_{k}\right)$. If $x_{k}=\bar{x}$ for infinitely many $k$ we have $\xi \in \hat{N}_{\Omega}(\bar{x})$ due to closedness of $\hat{N}_{\Omega}(\bar{x})$. On the other hand if $x_{k} \neq \bar{x}$ for infinitely many $k$, we set $t_{k}:=\left\|x_{k}-\bar{x}\right\|$ and $u_{k}:=\left(x_{k}-\bar{x}\right) /\left\|x_{k}-\bar{x}\right\|$ and by passing to a subsequence we assume $\left(t_{k}\right) \downarrow 0$ and $u_{k} \rightarrow u \in \mathbb{S}$. Since $x_{k}=\bar{x}+t_{k} u_{k} \in \Omega$ we conclude $\xi \in N_{\Omega}(\bar{x}, u)$ as well as $u \in T_{\Omega}(\bar{x})$, completing the proof.

Proposition 2.1 [24, Proposition 3.3] Let $\mathbb{R}^{n}$ be written as $\mathbb{R}^{n}=\mathbb{R}^{n_{1}} \times \ldots \times \mathbb{R}^{n_{l}}$ and for $x \in \mathbb{R}^{n}$ write $x=\left(x_{1}, \ldots, x_{l}\right)$ with $x_{i} \in \mathbb{R}^{n_{i}}$. Let $C_{i} \subset \mathbb{R}^{n_{i}}$ be closed for $i=1, \ldots, l$, set $C=C_{1} \times \ldots \times C_{l}$ and consider a point $\bar{x}=\left(\bar{x}_{1}, \ldots, \bar{x}_{l}\right) \in C$ and a direction $h=$ $\left(h_{1}, \ldots, h_{l}\right) \in \mathbb{R}^{n}$. Then

$$
N_{C}(\bar{x} ; h) \subset N_{C_{1}}\left(\bar{x}_{1} ; h_{1}\right) \times \ldots \times N_{C_{l}}\left(\bar{x}_{l} ; h_{l}\right) .
$$

For a multifunction $M: \mathbb{R}^{n} \rightrightarrows \mathbb{R}^{m}$ and a direction $(u, v) \in \mathbb{R}^{n} \times \mathbb{R}^{m}$, the limiting coderivative of $M$ in direction $(u, v)$ at $(\bar{x}, \bar{y}) \in \operatorname{gph} M$ is defined as the multifunction $D^{*} M((\bar{x}, \bar{y}) ;(u, v)): \mathbb{R}^{m} \rightrightarrows \mathbb{R}^{n}$ given by

$$
D^{*} M((\bar{x}, \bar{y}) ;(u, v))(\eta):=\left\{\xi \in \mathbb{R}^{n} \mid(\xi,-\eta) \in N_{\mathrm{gph} M}((\bar{x}, \bar{y}) ;(u, v))\right\} .
$$

Clearly, one has $D^{*} M((\bar{x}, \bar{y}) ;(0,0))=D^{*} M(\bar{x}, \bar{y})$. In case of a continuously differentiable single-valued mapping, the following relations hold.

Remark 2.1 Let $F: \mathbb{R}^{n} \rightarrow \mathbb{R}^{m}$ be continuously differentiable and let $\nabla F(\bar{x})$ denote its Jocobian. One has $D F(\bar{x})(u)=\nabla F(\bar{x}) u$ and thus $D^{*} F(\bar{x} ;(u, v))(\eta) \neq \varnothing$ if and only if $v=\nabla F(\bar{x}) u$, in which case

$$
D^{*} F(\bar{x} ;(u, v))(\eta)=D^{*} F(\bar{x})(\eta)=\hat{D}^{*} F(\bar{x})(\eta)=(\nabla F(\bar{x}))^{T} \eta .
$$

Our approach to directional limiting subdiferentials differs from the one established in [6, $14,19]$, where it is either defined or equivalently described as a limit of regular subdiferentials. In the finite dimensional setting these definitions read as follows. Given $f: \mathbb{R}^{n} \rightarrow \overline{\mathbb{R}}$, $\bar{x} \in \operatorname{dom} f$ and a direction $u \in \mathbb{R}^{n}$, consider the set

$\partial_{a} f(\bar{x}, u):=\left\{\xi \in \mathbb{R}^{n} \mid \exists\left(t_{k}\right) \downarrow 0,\left(u_{k}\right) \rightarrow u,\left(\xi_{k}\right) \rightarrow \xi: f\left(\bar{x}+t_{k} u_{k}\right) \rightarrow f(\bar{x}), \xi_{k} \in \hat{\partial} f\left(\bar{x}+t_{k} u_{k}\right) \forall k\right\}$, 
which we will call the analytic limiting subdiferential of $f$ at $\bar{x}$ in direction $u$, following the notation from [20, Definition 1.83]. ${ }^{1}$

In this paper, inspired by directional coderivatives, we consider a direction $(u, \mu) \in \mathbb{R}^{n+1}$ and define the limiting subdiferential of $f$ at $\bar{x}$ in direction $(u, \mu)$ via

$$
\partial f(\bar{x} ;(u, \mu)):=\left\{\xi \in \mathbb{R}^{n} \mid(\xi,-1) \in N_{\text {epi } f}((\bar{x}, f(\bar{x})) ;(u, \mu))\right\} .
$$

The advantages of this definition are twofold: First, it leads to a finer analysis and second, there is a close relationship between subdiferentials and normal cones which allows us to easily carry over the results obtained for normal cones to subdiferentials. More detailed discussion about the two versions of directional subdiferentials is presented at the beginning of Section 4.

Finally, we present some well-known properties of multifunctions as well as their directional counterparts. In order to do so, following [6], we define a directional neighborhood of (a direction) $u \in \mathbb{R}^{n}$.

Given a direction $u \in \mathbb{R}^{n}$ and positive numbers $\rho, \delta>0$, consider the set $\mathcal{V}_{\rho, \delta}(u)$ given by

$$
\mathcal{V}_{\rho, \delta}(u):=\{z \in \rho \mathbb{B} \mid\|\| u\|z-\| z\|u\| \leq \delta\|z\|\|u\|\} .
$$

We say that a set $\mathcal{V}$ is a directional neighborhood of $u$ if there exist $\rho, \delta>0$ such that $\mathcal{V}_{\rho, \delta}(u) \subset \mathcal{V}$. Moreover, we say that a sequence $x_{k} \in \mathbb{R}^{n}$ converges to some $\bar{x}$ from direction $u \in \mathbb{R}^{n}$ if for every directional neighborhood $\mathcal{V}$ of $u$ we have $x_{k} \in \bar{x}+\mathcal{V}$ for sufficiently large $k$, or, equivalently, if there exist $\left(t_{k}\right) \downarrow 0$ and $u_{k} \rightarrow u$ with $x_{k}=\bar{x}+t_{k} u_{k}$.

Definition 2.1 Let $M: \mathbb{R}^{n} \rightrightarrows \mathbb{R}^{m}$ and $(\bar{x}, \bar{y}) \in \operatorname{gph} M$. We say that $M$ is metrically subregular at $(\bar{x}, \bar{y})$ provided there exist $\kappa>0$ and a neighborhood $U$ of $\bar{x}$ such that

$$
\mathrm{d}_{M^{-1}(\bar{y})}(x) \leq \kappa \mathrm{d}_{M(x)}(\bar{y}) \forall x \in U .
$$

Given $u \in \mathbb{R}^{n}$, we say $M$ is metrically subregular in direction $u$ at $(\bar{x}, \bar{y})$ if there exists a directional neighborhood $\mathcal{U}$ of $u$ such that the above estimate holds for all $x \in \bar{x}+\mathcal{U}$.

We say that $S: \mathbb{R}^{m} \rightrightarrows \mathbb{R}^{n}$ is calm at $(\bar{y}, \bar{x}) \in \operatorname{gph} S$ provided there exist $\kappa>0$ and neighborhoods $U$ of $\bar{x}$ and $V$ of $\bar{y}$ such that

$$
S(y) \cap U \subset S(\bar{y})+\kappa\|y-\bar{y}\| \mathbb{B} \forall y \in V .
$$

It is known that neighborhood $U$ can be reduced (if necessary) in such a way that neighborhood $V$ can be replaced by the whole space $\mathbb{R}^{m}$, cf. [5, Exercise 3H.4].

For our purposes it is, however, suitable to employ estimate (4) without this simplification and to introduce the directional calmness by replacing $V$ by $\bar{y}+\mathcal{V}$, where $\mathcal{V}$ is the appropriate directional neighborhood.

While it is well-known that metric subregularity of $M$ at $(\bar{x}, \bar{y})$ is equivalent to calmness of $M^{-1}$ at $(\bar{y}, \bar{x})$, this does no longer hold for the directional versions. This is due to the fact that at directional metric subregularity the considered direction enters via neighborhood $U$ (Definition 2.1), whereas at directional calmness the directional neighborhood amounts to $V$, see (4).

Calmness, similarly as some other Lipschitzian stability properties enables us to estimate the images of $S$ around $(\bar{y}, \bar{x})$ via $S(\bar{y})$ and the respective calmness modulus $\kappa$. However,

\footnotetext{
${ }^{1}$ It was pointed out in [19] that in [6] the condition $f\left(\bar{x}+t_{k} u_{k}\right) \rightarrow f(\bar{x})$ was omitted from the definition and the same holds true for the definition from [14]. Such definition leads e.g. to violation of $\partial_{a} f(\bar{x}, 0)=\partial f(\bar{x})$, which is clearly undesirable and the omission of $f\left(\bar{x}+t_{k} u_{k}\right) \rightarrow f(\bar{x})$ was most likely unintentional.
} 
what we actually need for our directional calculus is the opposite, i.e., we need to be able to provide an estimate of $\bar{x}$ in terms of $S(y)$ for $y$ close to $\bar{y}$. This our need is reflected in the following inner version of calmness.

Definition 2.2 A set-valued mapping $S: \mathbb{R}^{m} \rightrightarrows \mathbb{R}^{n}$ is called inner calm at $(\bar{y}, \bar{x}) \in$ gph $S$ with respect to (w.r.t.) $\Omega \subset \mathbb{R}^{m}$ if there exist $\kappa>0$ and a neighborhood $V$ of $\bar{y}$ such that

$$
\bar{x} \in S(y)+\kappa\|y-\bar{y}\| \mathbb{B} \quad \forall y \in V \cap \Omega .
$$

If in the above definition $V=\bar{y}+\mathcal{V}$, where $\mathcal{V}$ is a directional neighborhood of a direction $v \in \mathbb{R}^{m}$, we say that $S$ possesses the inner calmness property at $(\bar{y}, \bar{x})$ w.r.t. $\Omega$ in direction $v$.

Note that inner calmness of $S$ at $(\bar{y}, \bar{x}) \in \operatorname{gph} S$ w.r.t. $\operatorname{dom} S$ in direction $v$ exactly corresponds to the directional inner semicompactness of $S$ at $(\bar{y}, \bar{x}) \in \operatorname{gph} S$ in direction $v$ from [19, Definition 4.4]. In literature one can find also several other names for this property, such as, e.g., Lipschitz lower semicontinuity [17] or recession with linear rate [15].

Apart from the notions of directional metric subregularity, calmness and inner calmness we will make use also of inner semicompactness and semicontinuity.

Recall that $S$ is inner semicompact at $\bar{y}$ w.r.t. $\Omega \subset \mathbb{R}^{m}$ if for every sequence $y_{k} \stackrel{\Omega}{\rightarrow} \bar{y}$ there exists a subsequence $K$ of $\mathbb{N}$ and a convergent sequence $\left(x_{k}\right)_{k \in K}$ with $x_{k} \in S\left(y_{k}\right)$ for $k \in K$. Given $\bar{x} \in S(\bar{y})$, we say that $S$ is inner semicontinuous at $(\bar{y}, \bar{x})$ w.r.t. $\Omega \subset \mathbb{R}^{m}$ if for every sequence $y_{k} \stackrel{\Omega}{\rightarrow} \bar{y}$ there exists a subsequence $K$ of $\mathbb{N}$ and a sequence $\left(x_{k}\right)_{k \in K}$ with $x_{k} \rightarrow \bar{x}$ and $x_{k} \in S\left(y_{k}\right)$ for $k \in K$. If $\Omega=\mathbb{R}^{m}$, we speak only about inner semicompactness at $\bar{y}$ and inner semicontinuity at $(\bar{y}, \bar{x})$. For more details we refer to [20].

The directional versions of inner semicompactness and semicontinuity are obtained by restricting our attention to $y_{k}$ converging to $\bar{y}$ from some direction $v$. We point out here that in [19, Definition 4.4] the authors defined the directional versions of inner semicompactness and semicontinuity in such a way that it allows them to find a suitable direction $h$, i.e., they control the rate of convergence $x_{k} \rightarrow \bar{x}$ by requiring the difference quotients $\left(x_{k}-\bar{x}\right) / t_{k}$ either to be bounded or to converge to some prescribed $h$. We believe, however, that it is not very suitable to call such properties semicompactness and semicontinuity, as those requirements are clearly much stronger and they are not implied by their non-directional counterparts, as also the authors admit.

Clearly, inner calmness implies both inner semicontinuity and semicompactness.

Interestingly, in [11, Theorem 8] Gfrerer and Outrata also investigated the estimate from definition of inner calmness and established some sufficient conditions to ensure both, calmness and inner calmness, of a class of solution maps.

Note that in case of a single-valued mapping $\varphi: \mathbb{R}^{m} \rightarrow \mathbb{R}^{n}$, calmness and inner calmness coincide and they read as

$$
\|\varphi(y)-\varphi(\bar{y})\| \leq \kappa\|y-\bar{y}\| \quad \forall y \in V .
$$

Further, $\varphi$ is called Lipschitz continuous near $\bar{y}$ in direction $v$ if the inequality

$$
\left\|\varphi(y)-\varphi\left(y^{\prime}\right)\right\| \leq \kappa\left\|y-y^{\prime}\right\| \forall y, y^{\prime} \in \bar{y}+\mathcal{V}
$$

is fulfilled with $\mathcal{V}$ being a directional neighborhood of $v$. Note that Lipschitz continuity of $\varphi$ near $\bar{y}$ in direction $v$ actually implies Lipschitz continuity of $\varphi$ near every point $y \in \bar{y}+\mathcal{V}$, $y \neq \bar{y}$.

In construction of the directional limiting calculus one is confronted with the following issue. Given a mapping $S: \mathbb{R}^{m} \rightrightarrows \mathbb{R}^{n}$, a point $\bar{y} \in \mathbb{R}^{m}$, a direction $v \in \mathbb{R}^{m}$ and a sequence $y_{k} \rightarrow \bar{y}$ from $v$ (i.e. $y_{k}=\bar{y}+t_{k} v_{k}$ for some $\left(t_{k}\right) \downarrow 0, v_{k} \rightarrow v$ ), we would like to identify 
not only a point $\bar{x} \in S(\bar{y})$ with $x_{k} \rightarrow \bar{x}$ for some $x_{k} \in S\left(y_{k}\right)$, but also a direction $h \in \mathbb{R}^{n}$ such that $x_{k}=\bar{x}+t_{k} h_{k}$ for some $h_{k} \rightarrow h$.

The task of finding an appropriate direction $h$ is related to the following sets. Given sequences $\left(a_{k}\right) \in \mathbb{R}^{n}$ and $\left(t_{k}\right) \downarrow 0$, we set

$\Gamma\left(a_{k}, t_{k}\right):=\left\{\omega \in \mathbb{R}^{n} \mid \exists\right.$ a subsequence $K$ of $\mathbb{N}: a_{k} / t_{k} \rightarrow \omega$ when $\left.k \in K\right\}$,

$\Gamma^{\infty}\left(a_{k}, t_{k}\right):=\left\{\omega \in \mathbb{S} \mid t_{k} /\left\|a_{k}\right\| \rightarrow 0, \exists\right.$ a subsequence $K$ of $\mathbb{N}: a_{k} /\left\|a_{k}\right\| \rightarrow \omega$ when $\left.k \in K\right\}$. (7)

Note that exactly one of these sets is not empty, since $\Gamma\left(a_{k}, t_{k}\right)=\emptyset$ is equivalent to $t_{k} /\left\|a_{k}\right\| \rightarrow 0$. In the situation considered above sequences $a_{k}$ appear in form $a_{k} \in$ $S\left(\bar{y}+t_{k} v_{k}\right)-S(\bar{y})$. Thus, if $\Gamma\left(a_{k}, t_{k}\right) \neq \varnothing$, one can clearly take a suitable direction $h \in \Gamma\left(a_{k}, t_{k}\right)$, while in the other case one can still proceed with $h \in \Gamma^{\infty}\left(a_{k}, t_{k}\right)$ to obtain different (but rather rough) estimates. Notation (6), (7) will be extensively used throughout the whole sequel.

Moreover, it is easy to see that inner calmness can be characterized in the following way.

Lemma 2.2 A set-valued mapping $S: \mathbb{R}^{m} \rightrightarrows \mathbb{R}^{n}$ is inner calm at $(\bar{y}, \bar{x}) \in$ gph $S$ w.r.t. $\Omega$ in direction $v$ if and only if for every $\left(t_{k}\right) \downarrow 0, v_{k} \rightarrow v$ with $\bar{y}+t_{k} v_{k} \in \Omega$ there exist a subsequence $K$ of $\mathbb{N}$ and a sequence $\left(x_{k}\right)_{k \in K}$ with $x_{k} \in S\left(\bar{y}+t_{k} v_{k}\right)$ for $k \in K$ such that $\Gamma\left(x_{k}-\bar{x}, t_{k}\right) \neq \emptyset$.

We conclude this preparatory section with a mention concerning qualification conditions used in the calculus being developed. Analogously to [16], our main qualification condition will be the directional metric subregularity of the so-called feasibility mapping associated with the considered calculus rule. This mapping has typically the form $F(x)=\Omega-\varphi(x)$, where $\Omega$ is a closed subset of $\mathbb{R}^{m}$ and $\varphi: \mathbb{R}^{n} \rightarrow \mathbb{R}^{m}$ is a continuous mapping. A tool for verifying directional metric subregularity of such mappings for continuously differentiable $\varphi$ was recently established by Gfrerer and Klatte [10, Corollary 1] and we slightly extend this result here by allowing functions $\varphi$ to be just calm in the prescribed direction.

Proposition 2.2 Let multifunction $F: \mathbb{R}^{n} \rightrightarrows \mathbb{R}^{m}$ be given by $F(x)=\Omega-\varphi(x)$, where $\varphi: \mathbb{R}^{n} \rightarrow \mathbb{R}^{m}$ is continuous and $\Omega \subset \mathbb{R}^{m}$ is a closed set. Further let $(\bar{x}, 0) \in$ gph $F$ and $u \in \mathbb{R}^{n}$ be given and assume that $\varphi$ is calm at $\bar{x}$ in direction $u$. Then $F$ is metrically subregular at $(\bar{x}, 0)$ in direction u provided for all $w \in D \varphi(\bar{x})(u) \cap T_{\Omega}(\varphi(\bar{x}))$ one has the implication

$$
0 \in D^{*} \varphi(\bar{x} ;(u, w))(\lambda), \lambda \in N_{\Omega}(\varphi(\bar{x}) ; w) \Longrightarrow \lambda=0 .
$$

The proof is based on the sum rule for coderivatives of multifunctions and will be presented among other applications in Section 6.

\section{Calculus for Directional Limiting Normal Cones}

Let $C \subset \mathbb{R}^{n}$ be a closed set and $\bar{x} \in C$. If $\bar{x} \in C \backslash$ bd $C$ then $N_{C}(\bar{x} ; h)=\{0\}$ for every $h \in \mathbb{R}^{n}$. Since $N_{C}(\bar{x} ; h)=\emptyset$ for $h \notin T_{C}(\bar{x})$ and $N_{C}(\bar{x} ; h)=\{0\}$ for $h \in T_{C}(\bar{x}) \backslash T_{\mathrm{bd} C}(\bar{x})$, it follows that for every set of directions $A \subset \mathbb{R}^{n}$ it holds that

$$
\bigcup_{h \in A} N_{C}(\bar{x} ; h)=\bigcup_{h \in A \cap T_{C}(\bar{x})} N_{C}(\bar{x} ; h)=\bigcup_{h \in A \cap T_{\mathrm{bd} C}(\bar{x})} N_{C}(\bar{x} ; h) .
$$


This observation allows us to consider only the indispensable directions in our estimates, as one can see in Theorems 3.1 and 3.2.

Theorem 3.1 (Pre-image sets) Let $Q \subset \mathbb{R}^{m}$ be closed, consider a continuous function $\varphi: \mathbb{R}^{n} \rightarrow \mathbb{R}^{m}$ and set $C:=\varphi^{-1}(Q)$. Assume further that the set-valued mapping $F:$ $\mathbb{R}^{n} \rightrightarrows \mathbb{R}^{m}$ given by $F(x)=Q-\varphi(x)$ is metrically subregular at $(\bar{x}, 0)$ in some direction $h \in \mathbb{R}^{n}$. Then

$$
\begin{array}{r}
N_{C}(\bar{x} ; h) \subset\left(\bigcup_{\substack{v \in D \varphi(\bar{x})(h) \\
\cap T_{Q}(\varphi(\bar{x}))}} D^{*} \varphi(\bar{x} ;(h, v)) N_{Q}(\varphi(\bar{x}) ; v)\right) \\
\cup\left(\bigcup_{\substack{v \in D \varphi(\bar{x})(0) \cap \mathbb{S} \\
\cap T_{Q}(\varphi(\bar{x}))}} D^{*} \varphi(\bar{x} ;(0, v)) N_{Q}(\varphi(\bar{x}) ; v)\right) .
\end{array}
$$

Moreover, if $\varphi$ is calm at $\bar{x}$ in direction $h$ we obtain a better estimate

$$
N_{C}(\bar{x} ; h) \subset \bigcup_{\substack{v \in D \varphi(\bar{x})(h) \\ \cap T_{Q}(\varphi(\bar{x}))}} D^{*} \varphi(\bar{x} ;(h, v)) N_{Q}(\varphi(\bar{x}) ; v) .
$$

Proof Let $x^{*} \in N_{C}(\bar{x} ; h)$ and consider $\left(t_{k}\right) \downarrow 0, h_{k} \rightarrow h, x_{k}^{*} \rightarrow x^{*}$ with $x_{k}:=\bar{x}+$ $t_{k} h_{k} \in C$ and $x_{k}^{*} \in \hat{N}_{C}\left(x_{k}\right)$. Since $x_{k}^{*} \in \hat{N}_{C}\left(x_{k}\right)$, for a fixed $k$ and for every $\varepsilon>0$ there exists a real $r_{\varepsilon}>0$ such that $\left\langle x_{k}^{*}, x-x_{k}\right\rangle \leq \varepsilon\left\|x-x_{k}\right\|$ for all $x \in C \cap \mathbb{B}_{r_{\varepsilon}}\left(x_{k}\right)$. Subregularity assumption yields existence of directional neighborhood $\mathcal{U}$ of $h$ and $\kappa>0$ such that $\mathrm{d}_{C}(x) \leq \kappa \mathrm{d}_{Q}(\varphi(x))$ holds for all $x \in \bar{x}+\mathcal{U}$ and for given sufficiently large $k$ and given $\varepsilon$ we choose $r_{\varepsilon}$ such that $\mathbb{B}_{r_{\varepsilon} / 2}\left(x_{k}\right) \subset \bar{x}+\mathcal{U}$.

Next we claim that for all $x \in \mathbb{B}_{r_{\varepsilon} / 2}\left(x_{k}\right)$ it holds that

$$
\varepsilon\left\|x-x_{k}\right\|-\left\langle x_{k}^{*}, x-x_{k}\right\rangle+\left(\left\|x_{k}^{*}\right\|+\varepsilon\right) \kappa \mathrm{d}_{Q}(\varphi(x)) \geq 0 .
$$

Indeed, for $x \in \mathbb{B}_{r_{\varepsilon} / 2}\left(x_{k}\right)$ we have $\left\|x-x_{k}\right\| \leq r_{\varepsilon} / 2$ and hence there exists $\tilde{x} \in C \cap \mathbb{B}_{r_{\varepsilon}}\left(x_{k}\right)$ with $\|x-\tilde{x}\|=\mathrm{d}_{C}(x)$. Thus,

$\left\langle x_{k}^{*}, x-x_{k}\right\rangle-\varepsilon\left\|x-x_{k}\right\| \leq\left(\left\|x_{k}^{*}\right\|+\varepsilon\right) \mathrm{d}_{C}(x)+\left\langle x_{k}^{*}, \tilde{x}-x_{k}\right\rangle-\varepsilon\left\|\tilde{x}-x_{k}\right\| \leq\left(\left\|x_{k}^{*}\right\|+\varepsilon\right) \kappa \mathrm{d}_{Q}(\varphi(x))$,

showing the claimed inequality.

Now we consider $\varepsilon_{k} \downarrow 0$ and conclude that $\left(x_{k}, \varphi\left(x_{k}\right), \varphi\left(x_{k}\right)\right)$ is a local solution of the problem

$\min f(x, y, q):=\varepsilon_{k}\left\|x-x_{k}\right\|-\left\langle x_{k}^{*}, x-x_{k}\right\rangle+\left(\left\|x_{k}^{*}\right\|+\varepsilon_{k}\right) \kappa\|y-q\|$ s.t. $(x, y, q) \in \operatorname{gph} \varphi \times Q$.

The fuzzy optimality conditions for problem (9), cf. [3, Theorem 2.7], [20, Lemma 2.32], state that to every $\eta_{k}>0$ there exist triples $\left(x_{i, k}, y_{i, k}, q_{i, k}\right) \in\left(x_{k}, \varphi\left(x_{k}\right), \varphi\left(x_{k}\right)\right)+\eta_{k} \mathbb{B}, i=$ 1,2 , with $\left|f\left(x_{1, k}, y_{1, k}, q_{1, k}\right)-f\left(x_{k}, \varphi\left(x_{k}\right), \varphi\left(x_{k}\right)\right)\right| \leq \eta_{k}$ and $\left(x_{2, k}, y_{2, k}, q_{2, k}\right) \in \operatorname{gph} \varphi \times Q$ such that

$$
\begin{gathered}
{\left[\begin{array}{l}
x_{k}^{*}-\varepsilon_{k} \xi_{k} \\
-\left(\left\|x_{k}^{*}\right\|+\varepsilon_{k}\right) \kappa v_{k}
\end{array}\right]} \\
\quad\left(\left\|x_{k}^{*}\right\|+\varepsilon_{k}\right) \kappa v_{k} \in \hat{N}_{\mathrm{gph} \varphi}\left(x_{2, k}, y_{2, k}\right)+\eta_{k} \mathbb{B},
\end{gathered}
$$

for some $\xi_{k}, v_{k} \in \mathbb{B}$. We take $\eta_{k}:=t_{k}^{2} \downarrow 0$ and consider the limiting process for $k \rightarrow \infty$. Since $\left(\left\|x_{k}^{*}\right\|+\varepsilon_{k}\right) \kappa v_{k}$ is a bounded sequence, by passing to a subsequence we can assume that $\left(\left\|x_{k}^{*}\right\|+\varepsilon_{k}\right) \kappa v_{k}$ converges to some $\bar{z}$. 
Denoting $a_{k}:=\varphi\left(\bar{x}+t_{k} h_{k}\right)-\varphi(\bar{x})$, we define direction $v$ to be an element of either $\Gamma\left(a_{k}, t_{k}\right)$ or $\Gamma^{\infty}\left(a_{k}, t_{k}\right)$, see (6)-(7). Let us first consider the case $v \in \Gamma\left(a_{k}, t_{k}\right)$ and assume $a_{k} / t_{k} \rightarrow v$. We show that $\bar{z} \in N_{Q}(\varphi(\bar{x}) ; v)$ and $x^{*} \in D^{*} \varphi(\bar{x} ;(h, v))(\bar{z})$. By virtue of (11) there is a sequence of vectors $z_{k} \in \hat{N}_{Q}\left(q_{2, k}\right)$ such that

$$
\left(\left\|x_{k}^{*}\right\|+\varepsilon_{k}\right) \kappa v_{k} \in z_{k}+\eta_{k} \mathbb{B} .
$$

Since $\eta_{k} \rightarrow 0$, we obtain $z_{k} \rightarrow \bar{z}$. Taking into account that, by virtue of the fuzzy optimality conditions,

$$
\left\|q_{2, k}-\varphi\left(x_{k}\right)\right\| \leq \eta_{k}=t_{k}^{2}
$$

we obtain

$$
\left\|\left(q_{2, k}-\varphi(\bar{x})\right) / t_{k}-v\right\| \leq\left\|\left(q_{2, k}-\varphi\left(x_{k}\right)\right) / t_{k}\right\|+\left\|\left(\varphi\left(\bar{x}+t_{k} h_{k}\right)-\varphi(\bar{x})\right) / t_{k}-v\right\| \rightarrow 0 .
$$

Since $\varphi(\bar{x})+t_{k}\left(q_{2, k}-\varphi(\bar{x})\right) / t_{k}=q_{2, k} \in Q$, the claimed relation $\bar{z} \in N_{Q}(\varphi(\bar{x}) ; v)$ follows.

In order to show the second claim, we observe that $x_{k}^{*}-\varepsilon_{k} \xi_{k} \rightarrow x^{*}$ and exploit in the same way as above relation (10) to show the existence of $\left(w_{k},-u_{k}\right) \in \hat{N}_{\mathrm{gph} \varphi}\left(x_{2, k}, y_{2, k}\right)$ with $\left(w_{k},-u_{k}\right) \rightarrow\left(x^{*},-\bar{z}\right)$. Again, one has that

$$
\left\|y_{2, k}-\varphi\left(x_{k}\right)\right\| \leq \eta_{k}=t_{k}^{2}
$$

and hence relation (12) holds with $q_{2, k}$ replaced by $y_{2, k}$. It follows that $\left(y_{2, k}-\varphi(\bar{x})\right) / t_{k} \rightarrow v$ and similarly we conclude also $\left(x_{2, k}-\bar{x}\right) / t_{k} \rightarrow h$. Thus, again, since $((\bar{x}, \varphi(\bar{x}))+$ $\left.t_{k}\left(x_{2, k}-\bar{x}\right) / t_{k},\left(y_{2, k}-\varphi(\bar{x})\right) / t_{k}\right)=\left(x_{2, k}, y_{2, k}\right) \in \operatorname{gph} \varphi$, we obtain $\left(x^{*},-\bar{z}\right) \in$ $N_{\operatorname{gph} \varphi}(\bar{x}, \varphi(\bar{x}) ;(h, v))$ and hence $x^{*} \in D^{*} \varphi(\bar{x} ;(h, v))(\bar{z})$ with $\bar{z} \in N_{Q}(\varphi(\bar{x}) ; v)$.

Finally, we consider the case $v \in \Gamma^{\infty}\left(a_{k}, t_{k}\right)$, assume $a_{k} /\left\|a_{k}\right\| \rightarrow v$ and show that $\bar{z} \in N_{Q}(\varphi(\bar{x}) ; v)$ and $x^{*} \in D^{*} \varphi(\bar{x} ;(0, v))(\bar{z})$. Note that in this case we have $v \in \mathbb{S}$ and $t_{k} /\left\|a_{k}\right\| \rightarrow 0$ implying $t_{k}<\left\|a_{k}\right\|$ for sufficiently large $k$. Hence, we proceed as in the previous case with $t_{k}$ replaced by $\left\|a_{k}\right\|$ and obtain the same result, the only difference being $\left(x_{2, k}-\bar{x}\right) /\left\|a_{k}\right\| \rightarrow 0$, showing the claimed relations. Observation (8) now completes the proof of the first statement.

The calmness assumption yields boundedness of $\left(\varphi\left(\bar{x}+t_{k} h_{k}\right)-\varphi(\bar{x})\right) / t_{k}$ and hence we always have $v \in \Gamma\left(a_{k}, t_{k}\right) \neq \varnothing$ and thus we only need to consider the first case. The proof is complete.

Let us illustrate Theorem 3.1 via the following simple example.

Example 3.1 Consider set $Q:=\mathbb{R}_{+}$and function $\varphi: \mathbb{R}^{2} \rightarrow \mathbb{R}$ given by

$$
\varphi(x):=\left\{\begin{aligned}
\sqrt{x_{1}}+x_{2} & \text { for } x_{1} \geq 0 \\
x_{2} & \text { otherwise. }
\end{aligned}\right.
$$

Note that $C:=\varphi^{-1}(Q)=\left(\mathbb{R}_{-} \times \mathbb{R}_{+}\right) \cup\left\{\left(x_{1}, x_{2}\right) \mid x_{1} \geq 0, x_{2} \geq-\sqrt{x_{1}}\right\}$ and let $\bar{x}:=(0,0)$. An easy computation yields that

$$
N_{C}(\bar{x})=N_{C}(\bar{x} ;(-1,0)) \cup N_{C}(\bar{x} ;(0,-1))=\left(\{0\} \times \mathbb{R}_{-}\right) \cup\left(\mathbb{R}_{-} \times\{0\}\right) .
$$


Let us take a look what estimates we obtain using Theorem 3.1. Directly from the definition we conclude that $Q-\varphi(x)$ is metrically subregular at $(\bar{x}, 0)$. Note that

$$
T_{\operatorname{gph} \varphi}(\bar{x}, \varphi(\bar{x}))=\{(a, b, b) \mid a<0\} \cup\{(0, b, c) \mid b \leq c\}
$$

and $T_{Q}(\varphi(\bar{x}))=Q=\mathbb{R}_{+}$. For the computation of the directional limiting normal cone to gph $\varphi$, consider arbitrary $a<0, b \in \mathbb{R}$ and $c>b$. We have to distinguish three cases:

$$
N_{\operatorname{gph} \varphi}((\bar{x}, \varphi(\bar{x})) ;(a, b, b))=\mathbb{R}(0,1,-1), N_{\operatorname{gph} \varphi}((\bar{x}, \varphi(\bar{x})) ;(0, b, c))=\mathbb{R}(1,0,0)
$$

and $N_{\operatorname{gph} \varphi}((\bar{x}, \varphi(\bar{x})) ;(0, b, b))=N_{\operatorname{gph} \varphi}(\bar{x}, \varphi(\bar{x}))=\mathbb{R}(0,1,-1) \cup \mathbb{R}(1,0,0) \cup\left(\mathbb{R}_{+} \times \mathbb{R}_{+}(1,-1)\right)$.

Consider now particular direction $\tilde{h}:=(0,-1)$. We have $D \varphi(\bar{x})(\tilde{h}) \cap T_{Q}(\varphi(\bar{x}))=\mathbb{R}_{+}$, $N_{Q}(\varphi(\bar{x}) ; \mu)=\{0\}$ and $D^{*} \varphi(\bar{x} ;(\tilde{h}, \mu))(0)=\mathbb{R}(1,0)$ for $\mu>0$ while $N_{Q}(\varphi(\bar{x}) ; 0)=\mathbb{R}_{-}$ and $D^{*} \varphi(\bar{x} ;(\tilde{h}, 0)) \mathbb{R}_{-}=\mathbb{R}(1,0)$. Moreover, $\varphi$ is not calm at $\bar{x}$ in direction $\tilde{h}$ and we thus proceed with the computation of $D \varphi(\bar{x})(0,0) \cap \mathbb{S}=\mathbb{R}_{+} \cap \mathbb{S}=\{1\}, N_{Q}(\varphi(\bar{x}) ; 1)=\{0\}$ and $D^{*} \varphi(\bar{x} ;(0,0,1))(0)=\mathbb{R}(1,0)$. Consequently, we obtain

$$
\begin{aligned}
& \mathbb{R}_{-} \times\{0\}=N_{C}(\bar{x} ; \tilde{h}) \\
& \subset \bigcup_{\mu \in \mathbb{R}_{+}} D^{*} \varphi(\bar{x} ;(\tilde{h}, \mu)) N_{Q}(\varphi(\bar{x}) ; \mu) \cup D^{*} \varphi(\bar{x} ;(0,0,1)) N_{Q}(\varphi(\bar{x}) ; 1)=\mathbb{R}(1,0) .
\end{aligned}
$$

Note that this shows that the both terms in Theorem 3.1 may be simultaneously nonempty.

Using similar arguments, it is not difficult to prove that, in fact, for every direction $0 \neq$ $\tilde{h}=\left(\tilde{h}_{1}, \tilde{h}_{2}\right) \in \mathbb{R}_{+} \times \mathbb{R}, \varphi$ fails to be calm at $\bar{x}$ in $\tilde{h}$ and Theorem 3.1 yields the estimate $N_{C}(\bar{x} ; \tilde{h}) \subset \mathbb{R}(1,0)$. In particular, if $\tilde{h}_{1}=0$, the both terms of the estimate are nonempty and coincide, whereas if $\tilde{h}_{1}>0$, the first union in Theorem 3.1 is taken over the empty set, demonstrating the need of the second term, since $N_{C}(\bar{x} ; \tilde{h})=\{(0,0)\} \neq \emptyset$.

On the other hand, for directions $\hat{h}=\left(\hat{h}_{1}, \hat{h}_{2}\right) \in(-\infty, 0) \times \mathbb{R}$, Theorem 3.1 actually provides exact estimates, namely $N_{C}(\bar{x} ; \hat{h})$ is subset of either $\emptyset$ if $\hat{h}_{2}<0$, or $\{0\} \times \mathbb{R}_{-}$if $\hat{h}_{2}=0$, or $\{(0,0)\}$ for $\hat{h}_{2}>0$.

Finally, for $h=(0,0)$ we obtain $N_{C}(\bar{x} ;(0,0))=N_{C}(\bar{x}) \subset\left(\{0\} \times \mathbb{R}_{-}\right) \cup \mathbb{R}(1,0)$.

Corollary 3.1 Let $C_{i} \subset \mathbb{R}^{n}, i=1, \ldots$, , be closed and set $C=\cap_{i=1}^{l} C_{i}$. Assume further that the set-valued mapping $F: \mathbb{R}^{n} \rightrightarrows \mathbb{R}^{n l}$ given by $F(x)=\prod_{i=1}^{l}\left(C_{i}-x\right)$ is metrically subregular at $(\bar{x},(0, \ldots, 0))$ in some direction $h \in \mathbb{R}^{n}$. Then

$$
N_{C}(\bar{x} ; h) \subset \sum_{i=1}^{l} N_{C_{i}}(\bar{x} ; h) .
$$

Proof Note that $C=\varphi^{-1}(Q)$ for $\varphi: \mathbb{R}^{n} \rightarrow \mathbb{R}^{n l}$ given by $\varphi(x):=(x, \ldots, x)(l$ copies $)$ and $Q:=\prod_{i=1}^{l} C_{i}$. Hence, the statement follows from Proposition 2.1, Theorem 3.1 and Remark 2.1.

Taking into account Proposition 2.2, as well as Remark 2.1 and Proposition 2.1, the assumption of metric subregularity of $F$ is implied by the condition

$$
\sum_{i=1}^{l} \lambda_{i}=0, \lambda_{i} \in N_{C_{i}}(\bar{x} ; h) \Longrightarrow \lambda_{i}=0 .
$$

Corollary 3.2 (Sets with constraint structure) Let $P \subset \mathbb{R}^{n}$ and $Q \subset \mathbb{R}^{m}$ be closed, consider a continuous function $\varphi: \mathbb{R}^{n} \rightarrow \mathbb{R}^{m}$ and set $C:=\{x \in P \mid \varphi(x) \in Q\}$. Assume further 
that the set-valued mapping $F: \mathbb{R}^{n} \rightrightarrows \mathbb{R}^{n} \times \mathbb{R}^{m}$ given by $F(x)=P \times Q-(x, \varphi(x))$ is metrically subregular at $(\bar{x}, 0,0)$ in some direction $h \in \mathbb{R}^{n}$. Then

$$
\begin{aligned}
N_{C}(\bar{x} ; h) \subset & \left(\bigcup_{\substack{v \in D \varphi(\bar{x})(h) \\
\cap T_{Q}(\varphi(\bar{x}))}} D^{*} \varphi(\bar{x} ;(h, v)) N_{Q}(\varphi(\bar{x}) ; v)+N_{P}(\bar{x} ; h)\right) \\
& \cup\left(\bigcup_{\substack{v \in D \varphi(\bar{x})(0) \cap \mathbb{S} \\
\cap T_{Q}(\varphi(\bar{x}))}} D^{*} \varphi(\bar{x} ;(0, v)) N_{Q}(\varphi(\bar{x}) ; v)+N_{P}(\bar{x} ; 0)\right) .
\end{aligned}
$$

Moreover, if $\varphi$ is calm at $\bar{x}$ in direction $h$ we obtain

$$
N_{C}(\bar{x} ; h) \subset \bigcup_{\substack{v \in D_{\varphi(\bar{x})(h)} \\ \cap T_{Q^{(\varphi(x))}}}} D^{*} \varphi(\bar{x} ;(h, v)) N_{Q}(\varphi(\bar{x}) ; v)+N_{P}(\bar{x} ; h) .
$$

Proof It is sufficient to apply Theorem 3.1 to the set $\tilde{C}=\Psi^{-1}(\tilde{Q})$ at $\bar{x}$ with $\Psi(x):=$ $(x, \varphi(x))$ and $\tilde{Q}=P \times Q$ and observe that $T_{\tilde{Q}}(\bar{x}, \varphi(\bar{x})) \subset T_{P}(\bar{x}) \times T_{Q}(\varphi(\bar{x}))([22$, Proposition 6.41]).

Assuming the calmness of $\varphi$ at $\bar{x}$ in direction $h$, Proposition 2.2 yields that the condition

$$
\lambda_{1} \in-D^{*} \varphi(\bar{x} ;(h, v))\left(\lambda_{2}\right) \cap N_{P}(\bar{x} ; h), \lambda_{2} \in N_{Q}(\varphi(\bar{x}) ; v) \Longrightarrow \lambda_{1}, \lambda_{2}=0,
$$

fulfilled for all $v \in D \varphi(\bar{x})(h) \cap T_{Q}(\varphi(\bar{x}))$, implies the required metric subregularity of $F$.

Theorem 3.2 (Image sets) Consider a closed set $C \subset \mathbb{R}^{n}$ and a continuous mapping $\varphi$ : $\mathbb{R}^{n} \rightarrow \mathbb{R}^{l}$, set $Q=\varphi(C)$ and consider $\bar{y} \in Q$ and a direction $v \in \mathbb{R}^{l}$. Let $\Psi: \mathbb{R}^{l} \rightrightarrows \mathbb{R}^{n}$ be given by $\Psi(y):=\varphi^{-1}(y) \cap C$. Assume that $\varphi: \mathbb{R}^{n} \rightarrow \mathbb{R}^{l}$ is Lipschitz continuous near every $\bar{x} \in \Psi(\bar{y})$ in all directions $h \in D \Psi(\bar{y}, \bar{x})(v)$ and $h \in D \Psi(\bar{y}, \bar{x})(0) \cap \mathbb{S}$. If $\Psi$ is inner semicompact at $\bar{y}$ w.r.t. $Q$ in direction $v$, then

$$
\begin{aligned}
N_{Q}(\bar{y} ; v) \subset \bigcup_{\bar{x} \in \Psi(\bar{y})} & \left(\left(\bigcup_{\substack{h \in\{\xi \mid v \in D \varphi(\bar{x})(\bar{\xi})\} \\
\cap T_{C}(\bar{x})}}\left\{y^{*} \mid D^{*} \varphi(\bar{x} ;(h, v))\left(y^{*}\right) \cap N_{C}(\bar{x} ; h) \neq \emptyset\right\}\right)\right. \\
& \left.\cup\left(\bigcup_{\substack{h \in\{\xi \in \mathbb{S} \mid 0 \in D \varphi(\bar{x})(\xi)\} \\
\cap T_{C}(\bar{x})}}\left\{y^{*} \mid D^{*} \varphi(\bar{x} ;(h, 0))\left(y^{*}\right) \cap N_{C}(\bar{x} ; h) \neq \emptyset\right\}\right)\right) .
\end{aligned}
$$

Moreover, if there exists $\bar{x} \in \Psi(\bar{y})$ such that $\Psi$ is inner semicontinuous at $(\bar{y}, \bar{x})$ w.r.t. $Q$ in direction $v$, then estimate (14) holds with this $\bar{x}$, i.e., the union over $\bar{x} \in \Psi(\bar{y})$ is superfluous. Finally, if there exists $\bar{x} \in \Psi(\bar{y})$ such that $\Psi$ is inner calm at $(\bar{y}, \bar{x})$ w.r.t. $Q$ in direction $v$, then (14) reduces to

$$
N_{Q}(\bar{y} ; v) \subset \bigcup_{\substack{h \in\{\xi \mid v \in D \varphi(\bar{x})(\xi)\} \\ \cap T_{C}(\bar{x})}}\left\{y^{*} \mid D^{*} \varphi(\bar{x} ;(h, v))\left(y^{*}\right) \cap N_{C}(\bar{x} ; h) \neq \emptyset\right\} .
$$

Proof Let $y^{*} \in N_{Q}(\bar{y} ; v)$ and consider $\left(t_{k}\right) \downarrow 0, v_{k} \rightarrow v, y_{k}^{*} \rightarrow y^{*}$ with $x_{k} \in \Psi\left(\bar{y}+t_{k} v_{k}\right)$ and $y_{k}^{*} \in \hat{N}_{Q}\left(\varphi\left(x_{k}\right)\right)$. Under the inner semicompactness of $\Psi$ we can take $x_{k} \in \Psi\left(\bar{y}+t_{k} v_{k}\right)$ converging to some $\bar{x} \in \Psi(\bar{y})$, taking also into account continuity of $\varphi$ and closedness of $C$. On the other hand, if we assume the existence of $\bar{x} \in \Psi(\bar{y})$ such that $\Psi$ is inner 
semicontinuous at $(\bar{y}, \bar{x})$ w.r.t. $Q$ in direction $v$, we can directly take $x_{k} \in \Psi\left(\bar{y}+t_{k} v_{k}\right)$ with $x_{k} \rightarrow \bar{x}$.

Now $y_{k}^{*} \in \hat{N}_{Q}\left(\varphi\left(x_{k}\right)\right)$ yields $\left\langle y_{k}^{*}, y-\varphi\left(x_{k}\right)\right\rangle \leq o\left(\left\|y-\varphi\left(x_{k}\right)\right\|\right)$ for all $y \in Q$. Thus, taking into account $\left\langle 0, x-x_{k}\right\rangle=0$ for all $x$ and $\left\|y-\varphi\left(x_{k}\right)\right\| \leq\left\|(y, x)-\left(\varphi\left(x_{k}\right), x_{k}\right)\right\|$, we obtain

$$
\left\langle\left(y_{k}^{*}, 0\right),(y, x)-\left(\varphi\left(x_{k}\right), x_{k}\right)\right\rangle \leq o\left(\left\|(y, x)-\left(\varphi\left(x_{k}\right), x_{k}\right)\right\|\right)
$$

for all $(y, x) \in \operatorname{gph} \Psi$, showing $\left(y_{k}^{*}, 0\right) \in \hat{N}_{\mathrm{gph} \Psi}\left(\varphi\left(x_{k}\right), x_{k}\right)$.

Denoting $a_{k}:=x_{k}-\bar{x}$, we define direction $h$ to be an element of either $\Gamma\left(a_{k}, t_{k}\right)$ or $\Gamma^{\infty}\left(a_{k}, t_{k}\right)$, see (6)-(7), and hence, taking also into account observation (8), we obtain

$$
\left(y^{*}, 0\right) \in\left(\bigcup_{h \in D \Psi(\bar{y}, \bar{x})(v)} N_{\mathrm{gph} \Psi}((\bar{y}, \bar{x}) ;(v, h))\right) \cup\left(\bigcup_{h \in D \Psi(\bar{y}, \bar{x})(0) \cap \mathbb{S}} N_{\mathrm{gph} \Psi}((\bar{y}, \bar{x}) ;(0, h))\right) .
$$

Note also that if $\Psi$ is inner calm at $(\bar{y}, \bar{x})$ w.r.t. $Q$ in direction $v$, it is also inner semicontinuous at $(\bar{y}, \bar{x})$ w.r.t. $Q$ in direction $v$ and there exists $x_{k} \in \Psi\left(\bar{y}+t_{k} v_{k}\right)$ such that $\left(x_{k}-\bar{x}\right) / t_{k}$ is bounded. Hence we always have $h \in \Gamma\left(a_{k}, t_{k}\right)$ and the second term in estimate (15) is superfluous.

Finally, gph $\Psi=\operatorname{gph} \varphi^{-1} \cap\left(\mathbb{R}^{l} \times C\right)$ and we can use Corollary 3.1. We consider only the case $\left(y^{*}, 0\right) \in \bigcup_{h \in D \Psi(\bar{y}, \bar{x})(v)} N_{\operatorname{gph} \Psi}((\bar{y}, \bar{x}) ;(v, h))$, because the other case is analogous. Note that condition (13) is fulfilled, because if $-\left(\lambda_{1}, \lambda_{2}\right) \in N_{\mathbb{R}^{l} \times C}((\bar{y}, \bar{x}) ;(v, h))$ we have $\lambda_{1}=0$ and $\left(0, \lambda_{2}\right) \in N_{\operatorname{gph} \varphi^{-1}}((\bar{y}, \bar{x}) ;(v, h))$ means $\lambda_{2} \in D^{*} \varphi(\bar{x} ;(h, v))(0)$, which implies $\lambda_{2}=0$ due to assumed Lipschitz continuity of $\varphi$ near $\bar{x}$ in direction $v$. Hence, taking into account Proposition 2.1, we obtain the existence of $h \in D \Psi(\bar{y}, \bar{x})(v),\left(w^{*}, z^{*}\right) \in$ $N_{\operatorname{gph} \varphi}((\bar{x}, \bar{y}) ;(h, v))$ and $x^{*} \in N_{C}(\bar{x} ; h)$ such that $\left(y^{*}, 0\right)=\left(z^{*}, w^{*}\right)+\left(0, x^{*}\right)$, i.e., $x^{*} \in$ $D^{*} \varphi(\bar{x} ;(h, v))\left(y^{*}\right) \cap N_{C}(\bar{x} ; h)$. This, together with (8), completes the proof.

Remark 3.1 Note that the directional Lipschitz continuity of $\varphi$ is only needed to justify the usage of Corollary 3.1. Thus, it can be weakened by assuming that for every $\bar{x} \in \Psi(\bar{y})$ and all directions $h \in D \Psi(\bar{y}, \bar{x})(v)$ and $h \in D \Psi(\bar{y}, \bar{x})(0) \cap \mathbb{S}$, the mapping

$$
(y, x) \rightrightarrows\left(\operatorname{gph} \varphi^{-1}-(y, x)\right) \times\left(\mathbb{R}^{l} \times C-(y, x)\right)
$$

is metrically subregular at $((\bar{y}, \bar{x}),(0,0,0,0))$ in directions $(v, h)$ and $(0, h)$.

Moreover, the inner semicompactness of $\Psi$ at $\bar{y}$ w.r.t. $Q$ in direction $v$ is clearly implied by the assumption that $\Psi((\bar{y}+\mathcal{V}) \cap Q)=\varphi^{-1}((\bar{y}+\mathcal{V}) \cap Q) \cap C$ is bounded for some directional neighborhood $\mathcal{V}$ of $v$. An analogous assumption was used in the standard version of this result in [22, Theorem 6.43].

Proposition 3.1 Let $C_{i} \subset \mathbb{R}^{n}, i=1, \ldots, l$, be closed and set $C=\cup_{i=1}^{l} C_{i}$. For $\bar{x} \in C$ and $h \in \mathbb{R}^{n}$ denote $I(\bar{x}):=\left\{i=1, \ldots, l \mid \bar{x} \in C_{i}\right\}$ and $I(\bar{x}, h):=\left\{i \in I(\bar{x}) \mid h \in T_{C_{i}}(\bar{x})\right\}$. Then

$$
N_{C}(\bar{x} ; h) \subset \bigcup_{i \in I(\bar{x}, h)} N_{C_{i}}(\bar{x} ; h)
$$

Proof Let $x^{*} \in N_{C}(\bar{x} ; h)$ and consider $\left(t_{k}\right) \downarrow 0, h_{k} \rightarrow h, x_{k}^{*} \rightarrow x^{*}$ with $\bar{x}+t_{k} h_{k} \in C$ and $x_{k}^{*} \in \hat{N}_{C}\left(\bar{x}+t_{k} h_{k}\right)$. Then there exists $i$ such that $\bar{x}+t_{k} h_{k} \in C_{i}$ for infinitely many $k$, showing $i \in I(\bar{x}, h)$. Since $C_{i} \subset C$, by passing to subsequence if necessary, we obtain $x_{k}^{*} \in \hat{N}_{C_{i}}\left(\bar{x}+t_{k} h_{k}\right)$, showing $x^{*} \in N_{C_{i}}(\bar{x} ; h)$. 


\section{Calculus for Directional Limiting Subdifferentials}

In this section we carry over the results for normal cones from the previous section to directional limiting subdifferentials defined via normals to the epigraph by (2). However, we start by a brief discussion about the relations between the analytic directional limiting subdifferential and the one given by (2).

Consider the following simple example.

Example 4.1 Let $\bar{x}=0, h=1$ and $f: \mathbb{R} \rightarrow \mathbb{R}$ be given by

$$
f(x)=\left\{\begin{aligned}
0 & \text { if } x \leq 0, \\
1 / \sqrt{k} & \text { if } x \in(1 /(k+1), 1 / k] \text { for } k \in \mathbb{N}, \\
1 & \text { if } x>1
\end{aligned}\right.
$$

Then clearly $f(x) \geq \sqrt{x}$ for $x \in[0,1]$ implying $D f(\bar{x})(h)=\emptyset$ and thus $\partial f(\bar{x} ;(h, v))=\emptyset$ for all $v$. On the other hand, $0 \in \hat{\partial} f(1 / k)$ for all $k \in \mathbb{N}$ and thus $0 \in \partial_{a} f(\bar{x} ; h)$.

In order to better understand the difference between the two concepts of directional subdifferentials, given an lsc function $f: \mathbb{R}^{n} \rightarrow \overline{\mathbb{R}}, \bar{x} \in \operatorname{dom} f$ and a direction $h \in \mathbb{R}^{n}$, we consider the following sets

$N_{\text {epi } f}((\bar{x}, f(\bar{x})) ;(h, \pm \infty)):=\left\{\left(x^{*}, \beta\right) \mid \exists\left(t_{k}\right) \downarrow 0,\left(h_{k}, v_{k}\right) \rightarrow(h, \pm \infty),\left(x_{k}^{*}, \beta_{k}\right) \rightarrow\left(x^{*}, \beta\right):\right.$

$$
\left.t_{k} v_{k} \rightarrow 0,\left(x_{k}^{*}, \beta_{k}\right) \in \hat{N}_{\text {epi } f}\left((\bar{x}, f(\bar{x}))+t_{k}\left(h_{k}, v_{k}\right)\right)\right\} \text {. }
$$

Proposition 4.1 Let $f: \mathbb{R}^{n} \rightarrow \overline{\mathbb{R}}$ be finite at $\bar{x}$ and lsc and consider $h \in \mathbb{R}^{n}$. One has

$$
\partial_{a} f(\bar{x} ; h)=\left\{x^{*} \mid\left(x^{*},-1\right) \in \bigcup_{v \in D f(\bar{x})(h)} N_{\text {epi } f}((\bar{x}, f(\bar{x})) ;(h, v)) \cup N_{\text {epif }}((\bar{x}, f(\bar{x})) ;(h, \pm \infty))\right\} .
$$

Proof Let $x^{*} \in \partial_{a} f(\bar{x} ; h)$ and consider $\left(t_{k}\right) \downarrow 0, h_{k} \rightarrow h, x_{k}^{*} \rightarrow x^{*}$ with $f\left(\bar{x}+t_{k} h_{k}\right) \rightarrow$ $f(\bar{x})$ and $x_{k}^{*} \in \hat{\partial} f\left(\bar{x}+t_{k} h_{k}\right)$, or, equivalently

$$
\left(x_{k}^{*},-1\right) \in \hat{N}_{\text {epi } f}\left(\bar{x}+t_{k} h_{k}, f\left(\bar{x}+t_{k} h_{k}\right)\right)=\hat{N}_{\text {epi } f}\left((\bar{x}, f(\bar{x}))+t_{k}\left(h_{k}, v_{k}\right)\right)
$$

for $\left.v_{k}:=\left(f\left(\bar{x}+t_{k} h_{k}\right)-f(\bar{x})\right) / t_{k}\right)$. Now if $v_{k} \rightarrow \pm \infty$, we immediately conclude $\left(x^{*},-1\right) \in N_{\text {epi } f}((\bar{x}, f(\bar{x})) ;(h, \pm \infty))$. On the other hand, if $v_{k} \nrightarrow \pm \infty$, there exists $v$ such that, after passing to a subsequence if necessary, we have $v_{k} \rightarrow v$. Thus, we conclude $\left(x^{*},-1\right) \in N_{\text {epi } f}((\bar{x}, f(\bar{x})) ;(h, v))$ and, taking into account $(\bar{x}, f(\bar{x}))+t_{k}\left(h_{k}, v_{k}\right) \in$ gph $f$, we obtain $v \in D f(\bar{x})(h)$.

Now take $x^{*}$ fulfilling $\left(x^{*},-1\right) \in N_{\text {epi } f}((\bar{x}, f(\bar{x})) ;(h, v))$ for some $v \in D f(\bar{x})(h)$ or $v= \pm \infty$ and consider $\left(t_{k}\right) \downarrow 0,\left(h_{k}, v_{k}\right) \rightarrow(h, v),\left(x_{k}^{*}, \beta_{k}\right) \rightarrow\left(x^{*},-1\right)$ with $(\bar{x}, f(\bar{x}))+$ $t_{k}\left(h_{k}, v_{k}\right) \in$ epi $f$ and $\left(x_{k}^{*}, \beta_{k}\right) \in \hat{N}_{\text {epi } f}\left((\bar{x}, f(\bar{x}))+t_{k}\left(h_{k}, v_{k}\right)\right)$. Since $f(\bar{x})+t_{k} v_{k}>$ $f\left(\bar{x}+t_{k} h_{k}\right)$ implies $\beta_{k}=0$, we assume that $f(\bar{x})+t_{k} v_{k}=f\left(\bar{x}+t_{k} h_{k}\right)$ and thus obtain $-x_{k}^{*} / \beta_{k} \in \hat{\partial} f\left(\bar{x}+t_{k} h_{k}\right)$ and consequently $x^{*} \in \partial_{a} f(\bar{x} ; h)$. Moreover, $f\left(\bar{x}+t_{k} h_{k}\right) \rightarrow f(\bar{x})$ follows directly from definition for $v= \pm \infty$ and for $v \in D f(\bar{x})(h)$ it holds due to lsc of $f$ and $(\bar{x}, f(\bar{x}))+t_{k}\left(h_{k}, v_{k}\right) \in$ epi $f$. This completes the proof.

Note that one always has

$$
N_{\text {epi } f}((\bar{x}, f(\bar{x})) ;(h, \pm \infty)) \subset N_{\text {epi } f}((\bar{x}, f(\bar{x})) ;(0, \pm 1))
$$


and this is due to the fact that for $f\left(x_{k}\right)-f(\bar{x})$ and $t_{k}$ from definition of $N_{\text {epi } f}((\bar{x}, f(\bar{x})) ;(h, \pm \infty))$ we have $\Gamma^{\infty}\left(f\left(x_{k}\right)-f(\bar{x}), t_{k}\right)= \pm 1$. Moreover, if $f$ is calm at $\bar{x}$ in direction $h$, the sequence $\left(f\left(x_{k}\right)-f(\bar{x})\right) / t_{k}$ is bounded and thus $N_{\text {epi } f}((\bar{x}, f(\bar{x})) ;(h, \pm \infty))=\emptyset$.

Note also that the calmness of an extended real-valued function is always understood with respect to its domain and hence does not exclude e.g. the indicator function of a set. Moreover, for our purposes, we in fact only need the existence of $\varepsilon, \kappa>0$ and a directional neighborhood $\mathcal{U}$ of $h$ such that

$$
|f(x)-f(\bar{x})| \leq \kappa\|x-\bar{x}\| \forall x \in \bar{x}+\mathcal{U} \text { with }|f(x)-f(\bar{x})| \leq \varepsilon,
$$

suggesting that discontinuities of $f$ also do not cause any harm. However, in order to keep the presentation as simple as possible, in the sequel we will only consider the calmness.

Corollary 4.1 Let $f: \mathbb{R}^{n} \rightarrow \overline{\mathbb{R}}$ be finite at $\bar{x}$ and consider $h \in \mathbb{R}^{n}$. Assume further that $f$ is calm at $\bar{x}$ in direction $h$. Then one has

$\partial_{a} f(\bar{x} ; h)=\bigcup_{v \in D f(\bar{x})(h)} \partial f(\bar{x} ;(h, v))=\left\{x^{*} \mid\left(x^{*},-1\right) \in \bigcup_{v \in D f(\bar{x})(h)} N_{e p i f}((\bar{x}, f(\bar{x})) ;(h, v))\right\}$.

If $f$ is also directionally differentiable at $\bar{x}$ in $h$, then $D f(\bar{x})(h)=f^{\prime}(\bar{x} ; h)$ and $\partial_{a} f(\bar{x} ; h)=\partial f\left(\bar{x} ;\left(h, f^{\prime}(\bar{x} ; h)\right)\right)$.

This corollary shows that the results for directional limiting subdifferentials obtained later in this section can be easily carried over to analytic directional limiting subdifferentials whenever the considered function $f$ is directionally calm. In the case when $f$ fails to be calm, one can get the results for analytic directional subdifferentials using the estimate

$$
\partial_{a} f(\bar{x} ; h) \subset \bigcup_{v \in D f(\bar{x})(h)} \partial f(\bar{x} ;(h, v)) \cup \partial f(\bar{x} ;(0,-1)) \cup \partial f(\bar{x} ;(0,1)),
$$

which follows from (17).

Another possible approach to calculus for directional limiting normal cones and subdifferentials would be to start with subdifferentials, build first the calculus for subdifferentials from the scratch and then carry it over to normal cones. The role of the bridge between the two concepts could be played by equivalent characterization of directional normal cones via directional subdifferentials of the indicator function or the distance function. For the sake of completeness, we present these results now.

Given a closed set $C$, we consider a point $\bar{x} \in C$ and a direction $h \in T_{C}(\bar{x})$. Clearly, $\delta_{C}(\cdot)$ and $\mathrm{d}_{C}(\cdot)$ are calm and directionally differentiable at $\bar{x}$ in $h$ with $\delta_{C}^{\prime}(\bar{x} ; h)=\mathrm{d}_{C}^{\prime}(\bar{x} ; h)=$ 0 . Thus, taking into account Corollary 4.1 , we can restrict our attention to the analytic subdifferentials.

While the relation $N_{C}(\bar{x}, h)=\partial_{a} \delta_{C}(\bar{x} ; h)$ follows directly from definitions, in order to deal with the distance function we need to consider the following lemma.

Lemma 4.1 For $C, \bar{x}$ and $h$ as above it holds that

$$
\partial_{a} \mathrm{~d}_{C}(\bar{x} ; h)=\left\{x^{*} \mid \exists\left(t_{k}\right) \downarrow 0, h_{k} \rightarrow h, x_{k}^{*} \rightarrow x^{*}: \bar{x}+t_{k} h_{k} \in C, x_{k}^{*} \in \hat{\partial} \mathrm{d}_{C}\left(\bar{x}+t_{k} h_{k}\right)\right\} .
$$

Proof Inclusion $\supset$ follows directly from definition. Now take $x^{*} \in \partial_{a} \mathrm{~d}_{C}(\bar{x} ; h)$ and consider $\left(t_{k}\right) \downarrow 0, \tilde{h}_{k} \rightarrow h, x_{k}^{*} \rightarrow x^{*}$ with $x_{k}^{*} \in \hat{\partial} \mathrm{d}_{C}\left(\bar{x}+t_{k} \tilde{h}_{k}\right)$. From [22, Example 8.53] we obtain 
$\hat{\partial} \mathrm{d}_{C}\left(\bar{x}+t_{k} \tilde{h}_{k}\right) \subset \hat{N}_{C}\left(x_{k}\right) \cap \mathbb{B}=\hat{\partial} \mathrm{d}_{C}\left(x_{k}\right)$ for every $x_{k} \in \mathrm{P}_{C}\left(\bar{x}+t_{k} \tilde{h}_{k}\right)$. Taking some $x_{k} \in \mathrm{P}_{C}\left(\bar{x}+t_{k} \tilde{h}_{k}\right)$ and setting $h_{k}:=\left(x_{k}-\bar{x}\right) / t_{k}$ we obtain $\bar{x}+t_{k} h_{k}=x_{k} \in C$. Moreover, since $h \in T_{C}(\bar{x})$ we conclude

$$
t_{k}\left\|h_{k}-\tilde{h}_{k}\right\|=\mathrm{d}_{C}\left(\bar{x}+t_{k} \tilde{h}_{k}\right) \leq \mathrm{d}_{C}\left(\bar{x}+t_{k} h\right)+t_{k}\left\|\tilde{h}_{k}-h\right\|=o\left(t_{k}\right),
$$

showing $h_{k} \rightarrow h$ and finishing the proof.

Corollary 4.2 Let $C \subset \mathbb{R}^{n}$ be a closed set, $\bar{x} \in C$ and $h \in T_{C}(\bar{x})$. Then

$$
N_{C}(\bar{x} ; h)=\bigcup_{\alpha>0} \alpha \partial_{a} \mathrm{~d}_{C}(\bar{x} ; h) .
$$

Proof Taking into account (19), the claim follows from [20, Corollary 1.96].

In [16], Ioffe and Outrata used subdifferentials of the distance function as the starting point for deriving the qualification conditions required for calculus rules. The previous lemma allows us to state a directional counterpart to their basic tool, [16, Proposition 3.1].

Corollary 4.3 Given $C, \bar{x}$ and $h$ as in the previous corollary, if $f: \mathbb{R}^{n} \rightarrow \overline{\mathbb{R}}$ is an lsc function fulfilling $f(x)=0, \forall x \in C$ and $f(x) \geq \mathrm{d}_{C}(x), \forall x \in \mathbb{R}^{n}$, then $\partial_{a} \mathrm{~d}_{C}(\bar{x} ; h) \subset$ $\partial_{a} f(\bar{x} ; h)$.

Proof The assumptions on $f$ imply $\hat{\partial} \mathrm{d}_{C}(x) \subset \hat{\partial} f(x)$ for every $x \in C$. Hence in (19) we obtain that $x_{k}^{*} \in \hat{\partial} f\left(\bar{x}+t_{k} h_{k}\right)$ and the claim follows.

Finally, given $f$ and $\bar{x}$ as before and a direction $(h, v) \in \mathbb{R}^{n+1}$, we introduce the singular subdifferential of $f$ at $\bar{x}$ in direction $(h, v)$ as

$$
\partial^{\infty} f(\bar{x} ;(h, v)):=\left\{x^{*} \in \mathbb{R}^{n} \mid\left(x^{*}, 0\right) \in N_{\text {epi } f}((\bar{x}, f(\bar{x})) ;(h, v))\right\} .
$$

This notion will be used in qualification conditions which mimic their counterparts from the "standard" generalized differential calculus. As it will be shown in Corollary 5.4 below, $\partial^{\infty} f(\bar{x} ;(h, v)) \subset\{0\}$ if $f$ is Lipschitz continuous near $\bar{x}$ in direction $h$.

\subsection{Chain rule and its corollaries}

We start this subsection by an auxiliary result concerning separable functions, which plays a role in deriving the sum rule from the chain rule. Note that, unlike the classical case [22, Proposition 10.5], we need to impose some mild assumptions in order to obtain a reasonable estimate.

Proposition 4.2 (Separable functions) Let $\mathbb{R}^{n}$ be decomposed as $\mathbb{R}^{n}=\mathbb{R}^{n_{1}} \times \ldots \times \mathbb{R}^{n_{l}}$ and let $x=\left(x_{1}, \ldots, x_{l}\right)$ with $x_{i} \in \mathbb{R}^{n_{i}}$. Let $f_{i}: \mathbb{R}^{n_{i}} \rightarrow \overline{\mathbb{R}}$ be lsc for $i=1, \ldots$, l and let all but one of $f_{i}$ be calm at $\bar{x}_{i}$ in direction $h_{i}$. Set $f(x)=f_{1}\left(x_{1}\right)+\ldots+f_{l}\left(x_{l}\right)$ and consider $\bar{x}=\left(\bar{x}_{1}, \ldots, \bar{x}_{l}\right) \in \operatorname{dom} f$ and some direction $(h, v)=\left(h_{1}, \ldots, h_{l}, v\right) \in \mathbb{R}^{n+1}$. Then

$$
\begin{aligned}
\partial f(\bar{x} ;(h, v)) & \subset \bigcup_{\substack{v_{i} \in D f_{i}\left(\bar{x}_{i}\right)\left(h_{i}\right) \\
v_{1}+\ldots+v_{l}=v}}\left(\partial f_{1}\left(\bar{x}_{1} ;\left(h_{1}, v_{1}\right)\right) \times \ldots \times \partial f_{l}\left(\bar{x}_{l} ;\left(h_{l}, v_{l}\right)\right)\right), \\
\partial^{\infty} f(\bar{x} ;(h, v)) \subset & \bigcup_{\substack{v_{i} \in D f_{i}\left(\bar{x}_{i}\right)\left(h_{i}\right) \\
v_{1}+\ldots+v_{l}=v}}\left(\partial^{\infty} f_{1}\left(\bar{x}_{1} ;\left(h_{1}, v_{1}\right)\right) \times \ldots \times \partial^{\infty} f_{l}\left(\bar{x}_{l} ;\left(h_{l}, v_{l}\right)\right)\right) .
\end{aligned}
$$


The proof follows from Theorem 3.2, since with $\varphi: \mathbb{R}^{n+l} \rightarrow \mathbb{R}^{n+1}$ given by $\varphi\left(x_{1}, \alpha_{1}, \ldots, x_{l}, \alpha_{l}\right):=\left(x_{1}, \ldots, x_{l}, \sum_{i=1}^{l} \alpha_{i}\right)$ we obtain epi $f=\varphi\left(\prod_{i=1}^{l}\right.$ epi $\left.f_{i}\right)$. Moreover, it can be shown that

$$
\Psi\left(x_{1}, \ldots, x_{l}, \alpha\right):=\left\{\left(x_{1}, \alpha_{1}, \ldots, x_{l}, \alpha_{l}\right) \in \prod_{i=1}^{l} \text { epi } f_{i} \mid \sum_{i=1}^{l} \alpha_{i}=\alpha\right\}
$$

is inner calm at $\left((\bar{x}, f(\bar{x})),\left(\bar{x}_{1}, f_{1}\left(\bar{x}_{1}\right), \ldots, \bar{x}_{l}, f_{l}\left(\bar{x}_{l}\right)\right)\right)$ w.r.t. epi $f$ in direction $(h, v)$, due to the calmness of all but one of $f_{i}$, even when the calmness is considered in the sense of (18). For the sake of brevity, the technical details are skipped.

Remark 4.1 Clearly, the calmness of all but one $f_{i}$ is just a sufficient condition that can be replaced by requiring the inner calmness of mapping (22). Moreover, one can also apply Theorem 3.2 without these assumptions to obtain more complicated estimates.

We have also stated the result concerning singular subdifferentials (21), because we will need it for deriving qualification conditions for the sum rule. Later on we will not write down the results for singular subdifferentials although usually the proofs will be applicable to this case as well.

Theorem 4.1 (Directional subdifferentials chain rule) Let $\varphi: \mathbb{R}^{n} \rightarrow \mathbb{R}^{m}$ be continuous, $g: \mathbb{R}^{m} \rightarrow \overline{\mathbb{R}}$ be finite at $\varphi(\bar{x})$ and lsc and set $f=g \circ \varphi$. Given a direction $(h, v) \in \mathbb{R}^{n+1}$, assume further that the set-valued mapping $F: \mathbb{R}^{n+1} \rightrightarrows \mathbb{R}^{m+1}$ given by $F(x, \alpha)=$ epi $g-$ $(\varphi(x), \alpha)$ is metrically subregular at $((\bar{x}, f(\bar{x})),(0,0))$ in direction $(h, v)$. Then

$$
\begin{aligned}
\partial f(\bar{x} ;(h, v)) \subset( & \left.\bigcup_{\substack{v \in\{w \in D \varphi(\bar{x})(h) \mid \\
v \in D(\varphi(\bar{x}))(w)\}}} D^{*} \varphi(\bar{x} ;(h, v)) \partial g(\varphi(\bar{x}) ;(v, v))\right) \\
& \cup\left(\bigcup_{\substack{v \in\{w \in D \varphi(\bar{x})(0) \cap \mathbb{S} \mid \\
0 \in D g(\varphi(\bar{x}))(w)\}}} D^{*} \varphi(\bar{x} ;(0, v)) \partial g(\varphi(\bar{x}) ;(v, 0))\right) .
\end{aligned}
$$

Moreover, if $\varphi$ is calm at $\bar{x}$ in direction $h$, then

$$
\partial f(\bar{x} ;(h, v)) \subset \bigcup_{\substack{v \in\{w \in D \varphi(\bar{x})(h)) \\ v \in D g(\varphi(\bar{x}))(w)\}}} D^{*} \varphi(\bar{x} ;(h, v)) \partial g(\varphi(\bar{x}) ;(v, v)) .
$$

Proof Take $\left(x^{*},-1\right) \in N_{\text {epi } f}((\bar{x}, f(\bar{x})) ;(h, v))$ and note that epi $f=\Psi^{-1}$ (epi $\left.g\right)$ for $\Psi(x, \alpha):=(\varphi(x), \alpha)$. Theorem 3.1 yields the existence of either $(v, \mu) \in$ $D \Psi(\bar{x}, f(\bar{x}))(h, v)$, such that

$$
\left(x^{*},-1\right) \in D^{*} \Psi((\bar{x}, f(\bar{x})) ;((h, v),(v, \mu))) N_{\text {epi } g}(\Psi(\bar{x}, f(\bar{x})) ;(v, \mu))
$$

or $(\tilde{v}, \tilde{\mu}) \in D \Psi(\bar{x}, f(\bar{x}))(0,0) \cap \mathbb{S}$ with

$$
\left(x^{*},-1\right) \in D^{*} \Psi((\bar{x}, f(\bar{x})) ;((0,0),(\tilde{v}, \tilde{\mu}))) N_{\text {epi } g}(\Psi(\bar{x}, f(\bar{x})) ;(\tilde{v}, \tilde{\mu})) .
$$

In the first case, there exists $\left(y^{*}, \beta\right) \in N_{\text {epi } g}(\Psi(\bar{x}, f(\bar{x})) ;(v, \mu))$ with

$$
\left(x^{*},-1,-y^{*},-\beta\right) \in N_{\mathrm{gph} \Psi}((\bar{x}, f(\bar{x}), \varphi(\bar{x}), f(\bar{x})) ;(h, v, v, \mu)) .
$$

Since $\operatorname{gph} \Psi=H(\operatorname{gph} \varphi \times \operatorname{gph} \mathrm{Id})$ for bijective function $H(x, y, a, b)=(x, a, y, b)$, applying Theorem 3.2 and Proposition 2.1 we obtain $\left(x^{*},-y^{*}\right) \in N_{\operatorname{gph} \varphi}((\bar{x}, \varphi(\bar{x})) ;(h, v))$ and $(-1,-\beta) \in N_{\mathrm{gph} I d}((f(\bar{x}), f(\bar{x})) ;(\nu, \mu))$. Note that the former relation also implies 
$v \in D \varphi(\bar{x})(h)$ and the latter yields $\beta=-1$ and $\mu=v$. Consequently $y^{*} \in \partial g(\varphi(\bar{x}) ;(v, v))$ and $v \in D g(\varphi(\bar{x}))(v)$.

On the other hand, in the case $(\tilde{v}, \tilde{\mu}) \in D \Psi(\bar{x}, f(\bar{x}))(0,0) \cap \mathbb{S}$ we can proceed analogously with replacing $(h, v)$ by $(0,0)$ to obtain $x^{*} \in D^{*} \varphi(\bar{x} ;(0, \tilde{v})) \partial g(\varphi(\bar{x}) ;(\tilde{v}, 0))$, $\tilde{v} \in D \varphi(\bar{x})(0), 0 \in D g(\varphi(\bar{x}))(\tilde{v})$ and $\tilde{\mu}=0$, showing also $\tilde{v} \in \mathbb{S}$.

Since the calmness of $\varphi$ at $\bar{x}$ in $h$ is equivalent to the calmness of $\Psi$ at $(\bar{x}, f(\bar{x}))$ in direction $(h, v)$, we obtain only the first possibility and hence the appropriate simpler estimate. This finishes the proof.

Taking into account Proposition 2.2 and the arguments from the proof and assuming the calmness of $\varphi$ at $\bar{x}$ in direction $h$, the condition

$$
0 \in D^{*} \varphi(\bar{x} ;(h, v))(\lambda), v \in D \varphi(\bar{x})(h), \lambda \in \partial^{\infty} g(\varphi(\bar{x}) ;(v, v)) \Longrightarrow \lambda=0
$$

implies the required directional metric subregularity of $F$.

Next consider $f: \mathbb{R}^{n} \times \mathbb{R}^{l} \rightarrow \overline{\mathbb{R}}$, a point $(\bar{x}, \bar{y}) \in \operatorname{dom} f$ and denote $f_{x}:=f(\cdot, \bar{y})$. Then, the partial subdifferential of $f$ with respect to $x$ at $\bar{x}$ for $\bar{y}$ in direction $(h, v)$ is given as $\partial_{x} f((\bar{x}, \bar{y}) ;(h, v)):=\partial f_{x}(\bar{x} ;(h, v))$.

Corollary 4.4 Let $f: \mathbb{R}^{n} \times \mathbb{R}^{l} \rightarrow \overline{\mathbb{R}}$ be finite at $(\bar{x}, \bar{y})$ and lsc. Given a direction $(h, v) \in$ $\mathbb{R}^{n+1}$, assume further that the set-valued mapping $F: \mathbb{R}^{n+1} \rightrightarrows \mathbb{R}^{n+l+1}$ given by $F(x, \alpha)=$ epi $f-(x, \bar{y}, \alpha)$ is metrically subregular at $((\bar{x}, f(\bar{x}, \bar{y})),(0,0,0))$ in $(h, v)$. Then

$$
\partial_{x} f((\bar{x}, \bar{y}) ;(h, v)) \subset\left\{x^{*} \mid \exists y^{*} \text { with }\left(x^{*}, y^{*}\right) \in \partial f((\bar{x}, \bar{y}) ;(h, 0, v))\right\} .
$$

Proof Since $f(\cdot, \bar{y})=f \circ \varphi$ with differentiable $\varphi(x)=(x, \bar{y})$, Theorem 4.1 yields the statement.

From Proposition 2.2 we infer that the above imposed metric subregularity of $F$ is implied by the condition

$$
(0, \lambda) \in \partial^{\infty} f((\bar{x}, \bar{y}) ;(h, 0, v)) \Longrightarrow \lambda=0 .
$$

Corollary 4.5 (Directional subdifferential sum rule) Suppose $f=f_{1}+\ldots+f_{l}$, where $f_{i}$ : $\mathbb{R}^{n} \rightarrow \overline{\mathbb{R}}$ are finite at $\bar{x}$, lsc and all but one are calm at $\bar{x}$ in direction $h \in \mathbb{R}^{n}$. Assume further that the set-valued mapping $F: \mathbb{R}^{n+1} \rightrightarrows \mathbb{R}^{n l+1}$ given by $F(x, \alpha)=$ epi $g-(x, \ldots, x, \alpha)$ with $g: \mathbb{R}^{n l} \rightarrow \overline{\mathbb{R}}$ defined via $g\left(x_{1}, \ldots, x_{l}\right)=f_{1}\left(x_{1}\right)+\ldots+f_{l}\left(x_{l}\right)$, is metrically subregular at $((\bar{x}, f(\bar{x})),(0, \ldots, 0,0))$ in direction $(h, v)$. Then

$$
\partial f(\bar{x} ;(h, v)) \subset \partial f_{1}(\bar{x} ;(h, v))+\ldots+\partial f_{l}(\bar{x} ;(h, v)) .
$$

Proof Note that $f=g \circ \varphi$ for $\varphi: \mathbb{R}^{n} \rightarrow \mathbb{R}^{n l}$ given by $\varphi(x):=(x, \ldots, x)$. Now the proof follows from Theorem 4.1, Proposition 4.2 and Remark 2.1.

Again, taking into account Propositions 2.2 and 4.2, the metric subregularity of $F$ from Corollary 4.5 is implied by the condition

$$
\sum_{i=1}^{l} \lambda_{i}=0, \lambda_{i} \in \partial^{\infty} f_{i}(\bar{x},(h, v)) \Longrightarrow \lambda_{i}=0 .
$$

Inclusion (23) holds true in particular if all but one of $f_{i}$ are Lipschitz continuous near $\bar{x}$ in direction $h$.

Naturally, Remark 4.1 applies here as well. 


\subsection{Directional limiting subdifferentials of special functions}

We conclude this section with estimates for directional limiting subdifferentials of the pointwise maximum and minimum of a finite family of functions, the distance function and the value function.

Proposition 4.3 Suppose $f=\max \left\{f_{1}, \ldots, f_{l}\right\}$ for functions $f_{i}: \mathbb{R}^{n} \rightarrow \overline{\mathbb{R}}$ that are continuous at $\bar{x}$. Given a direction $(h, v) \in \mathbb{R}^{n+1}$, assume further that the set-valued mapping $F: \mathbb{R}^{n+1} \rightrightarrows \mathbb{R}^{l(n+1)}$ given by $F(x, \alpha)=\prod_{i=1}^{l}\left(\right.$ epi $\left.f_{i}-(x, \alpha)\right)$ is metrically subregular at $((\bar{x}, f(\bar{x})),((0,0), \ldots,(0,0))$ in $(h, v)$. Then one has

$\partial f(\bar{x} ;(h, v)) \subset \bigcup_{J \subset I_{0}(\bar{x},(h, v))}\left(\operatorname{conv}\left\{\partial f_{i}(\bar{x} ;(h, v)) \mid i \in J\right\}+\sum_{i \in I(\bar{x},(h, v)) \backslash J} \partial^{\infty} f_{i}(\bar{x} ;(h, v))\right)$, where

$$
\begin{aligned}
I(\bar{x},(h, v)) & :=\left\{i \mid f(\bar{x})=f_{i}(\bar{x}) \text { and } v \in D f_{i}(\bar{x}) h\right\} \\
I_{0}(\bar{x},(h, v)) & :=\left\{i \in I(\bar{x},(h, v)) \mid \partial f_{i}(\bar{x} ;(h, v)) \neq \emptyset\right\} .
\end{aligned}
$$

Proof Since epi $f=\bigcap_{i=1}^{l}$ epi $f_{i}$, Corollary 3.1 yields that for $\left(x^{*},-1\right) \in$ $N_{\text {epi } f}((\bar{x}, f(\bar{x})),(h, v))$ there exists $\left(x_{i}^{*},-\beta_{i}\right) \in N_{\text {epi } f_{i}}((\bar{x}, f(\bar{x})),(h, v))$ such that $\left(x^{*},-1\right)=\sum_{i=1}^{l}\left(x_{i}^{*},-\beta_{i}\right)$. If $f_{i}(\bar{x})<f(\bar{x})$ for some $i$, we have $\left(x_{i}^{*},-\beta_{i}\right)=(0,0)$ due to continuity of $f_{i}$ and the same holds true if $v \notin D f_{i}(\bar{x}) h$. Hence we consider only $i \in I(\bar{x},(h, v))$.

Given a function $g$ and a point $x \in \operatorname{dom} g$, one always has that $\left(y^{*},-\beta\right) \in$ $\hat{N}_{\text {epi } g}(x, g(x))$ implies $\beta \geq 0$ and hence we obtain $\beta_{i} \geq 0$ for all $i \in I(\bar{x},(h, v))$. Setting $J:=\left\{i \in I(\bar{x},(h, v)) \mid \beta_{i}>0\right\}$, for $i \in J$ we obtain $\beta_{i}\left(x_{i}^{*} / \beta_{i},-1\right) \in$ $N_{\text {epi } f_{i}}\left(\left(\bar{x}, f_{i}(\bar{x})\right),(h, v)\right)$ and thus $x_{i}^{*} \in \beta_{i} \partial f_{i}(\bar{x} ;(h, v))$, showing also $J \subset I_{0}(\bar{x},(h, v))$. On the other hand, for $i \notin J$ we have $\beta_{i}=0$ and hence $x_{i}^{*} \in \partial^{\infty} f_{i}(\bar{x} ;(h, v))$. This completes the proof.

Taking into account (13) and the mentioned fact that $\left(y^{*},-\beta\right) \in \hat{N}_{\text {epi } g}(x, g(x))$ implies $\beta \geq 0$, the required metric subregularity of $F$ from Proposition 4.3 is again implied by condition (23).

Proposition 4.4 Suppose $f=\min \left\{f_{1}, \ldots, f_{l}\right\}$ for lsc functions $f_{i}: \mathbb{R}^{n} \rightarrow \overline{\mathbb{R}}$ and $\bar{x} \in$ dom $f$. Given a direction $(h, v) \in \mathbb{R}^{n+1}$, consider index set $I_{0}(\bar{x},(h, v))$ given by (24). Then one has

$$
\partial f(\bar{x} ;(h, v)) \subset \bigcup_{i \in I_{0}(\bar{x},(h, v))} \partial f_{i}(\bar{x} ;(h, v)) .
$$

Proof Since epi $f=\bigcup_{i=1}^{l}$ epi $f_{i}$, Proposition 3.1 yields that for $\left(x^{*},-1\right) \in$ $N_{\text {epi } f}((\bar{x}, f(\bar{x})),(h, v))$ there exists $i$ such that $\left(x^{*},-1\right) \in N_{\text {epi } f_{i}}((\bar{x}, f(\bar{x})),(h, v))$. Moreover, $(\bar{x}, f(\bar{x})) \in$ epi $f_{i}$ implies $f_{i}(\bar{x}) \leq f(\bar{x})$ while $f_{i}(\bar{x}) \geq f(\bar{x})$ follows from definition of $f$ and consequently $x^{*} \in \partial f_{i}(\bar{x} ; h)$. This verifies that $i \in I_{0}(\bar{x}, h)$ and completes the proof.

Theorem 4.2 (Directional subdifferentials of value function) Consider an lsc function $f$ : $\mathbb{R}^{n} \times \mathbb{R}^{l} \rightarrow \overline{\mathbb{R}}$, set $\vartheta(y)=\inf _{x \in \mathbb{R}^{n}} f(x, y)$ and assume that $\vartheta$ is finite at $\bar{y}$. Let $S: \mathbb{R}^{l} \rightrightarrows \mathbb{R}^{n}$ 
be the solution mapping given by $S(y)=\operatorname{argmin} f(\cdot, y)$ and consider a direction $(v, \mu) \in$ $\mathbb{R}^{l+1}$. If $S$ is inner semicompact at $\bar{y}$ in direction $v$, then

$$
\begin{aligned}
y^{*} \in \partial \vartheta(\bar{y} ;(v, \mu)) \Longrightarrow\left(0, y^{*}\right) \in \bigcup_{\bar{x} \in S(\bar{y})} & \left(\left(_{h \in\{\xi \mid \mu \in D f(\bar{x}, \bar{y})(\xi, v)\}} \partial f((\bar{x}, \bar{y}) ;(h, v, \mu))\right)\right. \\
& \left.\cup\left(\bigcup_{h \in\{\xi \in \mathbb{S} \mid 0 \in D f(\bar{x}, \bar{y})(\xi, 0)\}} \partial f((\bar{x}, \bar{y}) ;(h, 0,0))\right)\right) .
\end{aligned}
$$

Moreover, if there exists $\bar{x} \in S(\bar{y})$ such that $S$ is inner semicontinuous at $(\bar{y}, \bar{x})$ in direction $v$, then the previous estimate holds with this $\bar{x}$, i.e., the union over $\bar{x} \in S(\bar{y})$ is superfluous. Finally, if there exists $\bar{x} \in S(\bar{y})$ such that $S$ is inner calm at $(\bar{y}, \bar{x})$ in direction $v$, then the estimate reduces to

$$
y^{*} \in \partial \vartheta(\bar{y} ;(v, \mu)) \Longrightarrow\left(0, y^{*}\right) \in \bigcup_{h \in\{\xi \mid \mu \in D f(\bar{x}, \bar{y})(\xi, v)\}} \partial f((\bar{x}, \bar{y}) ;(h, v, \mu)) .
$$

Proof Let $\left(y^{*},-1\right) \in N_{\text {epi } \vartheta}(\bar{y}, \vartheta(\bar{y}) ;(v, \mu))$. The assumptions imposed on $S$ imply that $S(y)$ is locally not empty-valued (around $\bar{y}$ ). Hence, we may proceed as in [22, Theorem $10.12]$ to obtain epi $\vartheta=\varphi($ epi $f)$ with $\varphi: \mathbb{R}^{n+l+1} \rightarrow \mathbb{R}^{l+1}$ given by $\varphi(x, y, \alpha)=(y, \alpha)$. In order to apply Theorem 3.2 we yet have to show that the assumptions on $S$ imply the corresponding assumptions on $\Psi(y, \alpha):=\{(x, y, \alpha) \mid(x, y, \alpha) \in$ epi $f\}$ w.r.t. epi $\vartheta$. Note that if $(y, \alpha) \in$ epi $\vartheta$ and $x \in S(y)$ we have $\alpha \geq \vartheta(y)=f(x, y)$ and hence $\{(x, y, \alpha) \mid(y, \alpha) \in$ epi $\vartheta, x \in S(y)\} \subset \Psi(y, \alpha)$.

Consider $\left(y_{k}, \alpha_{k}\right) \stackrel{\text { epi } \vartheta}{\rightarrow}(\bar{y}, \vartheta(\bar{y}))$ from direction $(v, \mu)$ and thus $y_{k} \rightarrow \bar{y}$ from direction $v$. The inner semicompactness of $S$ yields the existence of $\bar{x}$ and a sequence $x_{k} \rightarrow \bar{x}$ such that, by passing to a subsequence, we have $x_{k} \in S\left(y_{k}\right)$ and hence $\left(x_{k}, y_{k}, \alpha_{k}\right) \in \Psi\left(y_{k}, \alpha_{k}\right)$ with $\left(x_{k}, y_{k}, \alpha_{k}\right) \rightarrow(\bar{x}, \bar{y}, \vartheta(\bar{y}))$, showing the inner semicompactness of $\Psi$ at $(\bar{y}, \vartheta(\bar{y}))$ w.r.t. epi $\vartheta$ in direction $(v, \mu)$.

Now fix $\bar{x} \in S(\bar{y})$. If $S$ is inner semicontinuous at $(\bar{y}, \bar{x})$ in $v$, the inner semicontinuity of $\Psi$ at $((\bar{y}, \vartheta(\bar{y})),(\bar{x}, \bar{y}, \vartheta(\bar{y})))$ w.r.t. epi $\vartheta$ in $(v, \mu)$ follows from analogous arguments. Assuming the inner calmness of $S$, let $\mathcal{V}$ denote the directional neighborhood of $v$ such that $\bar{x} \in S(y)+L\|y-\bar{y}\|$ for all $y \in \bar{y}+\mathcal{V}$ and consider a directional neighborhood $\mathcal{W}$ of $(v, \mu)$ such that for $(y, \alpha) \in((\bar{y}, \vartheta(\bar{y}))+\mathcal{W}) \cap$ epi $\vartheta$ we have $y \in \bar{y}+\mathcal{V}$. We obtain that there exists $x \in S(y)$, i.e., $(x, y, \alpha) \in \Psi(y, \alpha)$ such that

$$
\begin{aligned}
\|(\bar{x}, \bar{y}, \vartheta(\bar{y}))-(x, y, \alpha)\| & \leq\|\bar{x}-x\|+\|(\bar{y}, \vartheta(\bar{y}))-(y, \alpha)\| \\
& \leq L\|y-\bar{y}\|+\|(\bar{y}, \vartheta(\bar{y}))-(y, \alpha)\| \\
& \leq(L+1)\|(\bar{y}, \vartheta(\bar{y}))-(y, \alpha)\|,
\end{aligned}
$$

showing the inner calmness of $\Psi$ at $((\bar{y}, \vartheta(\bar{y})),(\bar{x}, \bar{y}, \vartheta(\bar{y})))$ w.r.t. epi $\vartheta$ in $(v, \mu)$.

Taking into account the differentiability of $\varphi$, Theorem 3.2 now yields all statements of the theorem.

\section{Calculus for Directional Coderivatives}

In the first part of this section we present two basic calculus rules, namely the chain rule and the sum rule for directional limiting coderivatives. In fact, having proved one of them, the other one can be derived relatively easily on the basis of the first one, similarly like 
in the case of standard limiting coderivatives. Here we follow essentially the pattern from [22]. Thereafter we present a "scalarization" formula which may facilitate the computation of coderivatives of single-valued Lipschitz continuous mappings.

Consider first the mappings $S_{1}: \mathbb{R}^{n} \rightrightarrows \mathbb{R}^{m}, S_{2}: \mathbb{R}^{m} \rightrightarrows \mathbb{R}^{s}$ and associate with them the "intermediate" multifunction $\Xi: \mathbb{R}^{n} \times \mathbb{R}^{s} \rightrightarrows \mathbb{R}^{m}$ defined by

$$
\Xi(x, u)=\left\{w \in S_{1}(x) \mid u \in S_{2}(w)\right\} .
$$

Theorem 5.1 (Directional coderivative chain rule) Suppose $S=S_{2} \circ S_{1}$ for osc mappings $S_{1}, S_{2}$. Let $\bar{x} \in \operatorname{dom} S, \bar{u} \in S(\bar{x})$ and $(h, l) \in \mathbb{R}^{n} \times \mathbb{R}^{s}$ be two given directions. Assume that

(a) there is a directional neighborhood $\mathcal{U}$ of $(h, l)$ such that $\Xi((\bar{x}, \bar{u})+\mathcal{U})$ is bounded;

(b) the mapping

$$
F(x, w, u):=\left[\begin{array}{l}
g p h S_{1}-(x, w) \\
g p h S_{2}-(w, u)
\end{array}\right]
$$

is metrically subregular at $(\bar{x}, w, \bar{u}, 0,0)$ for all $w \in \Xi(\bar{x}, \bar{u})$ in directions $(h, k, l)$ with $k$ such that $(h, k) \in T_{g p h S_{1}}(\bar{x}, w),(k, l) \in T_{g p h S_{2}}(w, \bar{u})$, and in directions $(0, k, 0)$ with $k \in \mathbb{S}$ such that $(0, k) \in T_{g p h S_{1}}(\bar{x}, w),(k, 0) \in T_{g p h S_{2}}(w, \bar{u})$;

Then one has

$$
\begin{aligned}
& D^{*} S((\bar{x}, \bar{u}) ;(h, l)) \subset \bigcup_{\tilde{w} \in \Xi(\bar{x}, \bar{u})}\left(\bigcup_{\substack{k \in\left\{\xi \in D S_{1}(\bar{x}, \tilde{w})(h) \mid \\
l \in D S_{2}(\tilde{w}, \bar{u})(\xi)\right\}}} D^{*} S_{1}((\bar{x}, \tilde{w}) ;(h, k)) \circ D^{*} S_{2}((\tilde{w}, \bar{u}) ;(k, l))\right. \\
& \left.\cup \quad \bigcup_{k \in\left\{\xi \in \mathbb{S} \mid \xi \in D S_{1}(\bar{x}, \tilde{w})(0),\right.} D^{*} S_{1}((\bar{x}, \tilde{w}) ;(0, k)) \circ D^{*} S_{2}((\tilde{w}, \bar{u}) ;(k, 0))\right) \text {. }
\end{aligned}
$$

Proof Following the proof idea of [22, Thm.10.37] one has that gph $S=G(C)$ with $G$ : $(x, w, u) \mapsto(x, u)$ and $C=H^{-1}(D)$, where $H:(x, w, u) \mapsto(x, w, w, u)$ and $D=$ gph $S_{1} \times \operatorname{gph} S_{2}$.

To compute an estimate of $N_{\mathrm{gph} S}((\bar{x}, \bar{u}) ;(h, l))$, we invoke first Theorem 3.2, which is possible thanks to condition (a), see also Remark 3.1. We obtain that

$$
\begin{aligned}
& N_{\operatorname{gph} S}((\bar{x}, \bar{u}) ;(h, l)) \subset \bigcup_{\tilde{w} \in \Xi(\bar{x}, \bar{u})}\left(\bigcup_{k \in\left\{\xi \mid(h, \xi, l) \in T_{C}(\bar{x}, \tilde{w}, \bar{u})\right\}}\left\{\left(y_{1}^{*}, y_{2}^{*}\right) \mid\left(y_{1}^{*}, 0, y_{2}^{*}\right) \in N_{C}((\bar{x}, \tilde{w}, \bar{u}) ;(h, k, l))\right\}\right. \\
& \left.\cup \bigcup_{k \in\left\{\xi \in \mathbb{S} \mid(0, \xi, 0) \in T_{C}(\bar{x}, \tilde{w}, \bar{u})\right\}}\left\{\left(y_{1}^{*}, y_{2}^{*}\right) \mid\left(y_{1}^{*}, 0, y_{2}^{*}\right) \in N_{C}((\bar{x}, \tilde{w}, \bar{u}) ;(0, k, 0))\right\}\right) .
\end{aligned}
$$

Next we compute $N_{C}((\bar{x}, \tilde{w}, \bar{u}) ;(h, k, l))$ via Theorem 3.1. Thanks to condition (b) and the calmness of $H$ one has

$$
\begin{aligned}
& N_{C}((\bar{x}, \tilde{w}, \bar{u}) ;(h, k, l)) \subset \\
& \left\{(a, b+c, d) \mid(a, b) \in N_{\operatorname{gph} S_{1}}((\bar{x}, \tilde{w}) ;(h, k)),(c, d) \in N_{\operatorname{gph} S_{2}}((\tilde{w}, \bar{u}) ;(k, l))\right\}
\end{aligned}
$$

and, likewise,

$$
\begin{aligned}
& N_{C}((\bar{x}, \tilde{w}, \bar{u}) ;(0, k, 0)) \subset \\
& \left\{(a, b+c, d) \mid(a, b) \in N_{\mathrm{gph} S_{1}}((\bar{x}, \tilde{w}) ;(0, k)),(c, d) \in N_{\mathrm{gph} S_{2}}((\tilde{w}, \bar{u}) ;(k, 0))\right\} .
\end{aligned}
$$

Further we observe that

$$
T_{C}(\bar{x}, \tilde{w}, \bar{u}) \subset\left\{(h, k, l) \mid(h, k) \in T_{\operatorname{gph} S_{1}}(\bar{x}, \tilde{w}),(k, l) \in T_{\operatorname{gph} S_{2}}(\tilde{w}, \bar{u})\right\},
$$


so that the first union in (27) with respect to $k$ can be taken over the set

$$
k \in\left\{\xi \mid(h, \xi) \in T_{\operatorname{gph} S_{1}}(\bar{x}, \tilde{w}),(\xi, l) \in T_{\operatorname{gph~} S_{2}}(\tilde{w}, \bar{u})\right\}=: \mathcal{T}_{h: l},
$$

and the second union in (27) with respect to $k$ can be taken over the set

$$
k \in\left\{\xi \in \mathbb{S} \mid(0, \xi) \in T_{\operatorname{gph} S_{1}}(\bar{x}, \tilde{w}),(\xi, 0) \in T_{\operatorname{gph} S_{2}}(\tilde{w}, \bar{u})\right\}=: \mathcal{T}_{0: 0}^{\mathbb{S}} .
$$

Using consecutively representations (30), (31) and inclusions (28), (29) we obtain that

$$
\begin{aligned}
& N_{\mathrm{gph} S}((\bar{x}, \bar{u}) ;(h, l)) \\
& \subset \bigcup_{\tilde{w} \in \Xi(\bar{x}, \bar{u})}\left\{\left(y_{1}^{*}, y_{2}^{*}\right) \mid\left(y_{1}^{*}, 0, y_{2}^{*}\right) \in \bigcup_{k \in \mathcal{T}_{h: l}} N_{C}((\bar{x}, \tilde{w}, \bar{u}) ;(h, k, l)) \cup \bigcup_{k \in \mathcal{T}_{0: 0}^{\mathbb{S}}} N_{C}((\bar{x}, \tilde{w}, \bar{u}) ;(0, k, 0))\right\} \\
& \subset \bigcup_{\tilde{w} \in \Xi(\bar{x}, \bar{u})}\left(\bigcup_{k \in \mathcal{T}_{h: l}}\left\{\left(y_{1}^{*}, y_{2}^{*}\right) \mid \exists c:\left(y_{1}^{*},-c\right) \in N_{\mathrm{gph} S_{1}}((\bar{x}, \tilde{w}) ;(h, k)),\left(c, y_{2}^{*}\right) \in N_{\operatorname{gph} S_{2}}((\tilde{w}, \bar{u}) ;(k, l))\right\}\right. \\
& \left.\quad \cup \bigcup_{k \in \mathcal{T}_{0: 0}^{\mathbb{S}}}\left\{\left(y_{1}^{*}, y_{2}^{*}\right) \mid \exists c:\left(y_{1}^{*},-c\right) \in N_{\operatorname{gph} S_{1}}((\bar{x}, \tilde{w}) ;(0, k)),\left(c, y_{2}^{*}\right) \in N_{\operatorname{gph} S_{2}}((\tilde{w}, \bar{u}) ;(k, 0))\right\}\right) .
\end{aligned}
$$

It follows that for $u^{*}:=-y_{2}^{*}$ one has

$$
\begin{aligned}
D^{*} S((\bar{x}, \bar{u}) ;(h, l))\left(u^{*}\right) & \subset \bigcup_{\tilde{w} \in \Xi(\bar{x}, \bar{u})}\left(\bigcup_{k \in \mathcal{T}_{h: l}} D^{*} S_{1}((\bar{x}, \tilde{w}) ;(h, k)) \circ D^{*} S_{2}((\tilde{w}, \bar{u}) ;(k, l))\left(u^{*}\right)\right. \\
& \left.\cup \bigcup_{k \in \mathcal{T}_{0: 0}^{\mathbb{S}}} D^{*} S_{1}((\bar{x}, \tilde{w}) ;(0, k)) \circ D^{*} S_{2}((\tilde{w}, \bar{u}) ;(k, 0))\left(u^{*}\right)\right),
\end{aligned}
$$

and the proof is complete.

Let us comment on assumption (b) which is, admittedly, not easy to verify in general. Following Proposition 2.1, it may be ensured by the next two conditions (which are, however, more restrictive).

(i) For all $\tilde{w} \in \Xi(\bar{x}, \bar{u})$ and all directions $k$ such that $k \in D S_{1}(\bar{x}, \tilde{w})(h), l \in$ $D S_{2}(\tilde{w}, \bar{u})(k)$ one has

$$
0 \in D^{*} S_{1}((\bar{x}, \tilde{w}) ;(h, k))(-\lambda), \lambda \in D^{*} S_{2}((\tilde{w}, \bar{u}) ;(k, l))(0) \quad \Longrightarrow \quad \lambda=0 ;
$$

(ii) for all $\tilde{w} \in \Xi(\bar{x}, \bar{u})$ and all directions $k \in \mathbb{S}$ such that $k \in D S_{1}(\bar{x}, \tilde{w})(0), 0 \in$ $D S_{2}(\tilde{w}, \bar{u})(k)$ one has

$$
0 \in D^{*} S_{1}((\bar{x}, \tilde{w}) ;(0, k))(-\lambda), \lambda \in D^{*} S_{2}((\tilde{w}, \bar{u}) ;(k, 0))(0) \quad \Longrightarrow \quad \lambda=0 .
$$

We observe that both conditions (32), (33) are automatically fulfilled provided either $S_{1}$ is metrically regular around $(\bar{x}, \tilde{w})$ for $\tilde{w} \in \Xi(\bar{x}, \bar{u})$, or $S_{2}$ has the Aubin property around $(\tilde{w}, \bar{u})$ for $\tilde{w} \in \Xi(\bar{x}, \bar{u})$. This complies with the corresponding conditions in [22, Theorem 10.37]. More precisely, one can employ the characterizations of the directional metric regularity and the Aubin property from [10, Theorem 1].

Further, on the basis of Theorem 3.2, note that the statement of Theorem 5.1 remains true if we weaken assumption (a) by asking only that the intermediate mapping $\Xi$ is inner semicompact at $(\bar{x}, \bar{u})$ (w.r.t. gph $S)$ in direction $(h, l)$. On the other hand, if we strengthen assumption (a) by asking that there exists some $\tilde{w} \in \Xi(\bar{x}, \bar{u})$ such that $\Xi$ is inner semicontinuous at $(\bar{x}, \bar{u}, \tilde{w})$ (w.r.t. gph $S)$ in direction $(h, l)$, then the inclusion (26) holds with this particular $\tilde{w}$ making the union over $\Xi(\bar{x}, \bar{u})$ superfluous. On top of that, the union over $k \in\left\{\xi \in \mathbb{S} \mid \xi \in D S_{1}(\bar{x}, \tilde{w})(0), 0 \in D S_{2}(\tilde{w}, \bar{u})(\xi)\right\}$ vanishes provided we assume that $\Xi$ is even inner calm at $(\bar{x}, \bar{u}, \tilde{w})$ (w.r.t. gph $S)$ in direction $(h, l)$. 
On the basis of the above considerations we obtain immediately the following corollaries of Theorem 5.1 .

Corollary 5.1 In the framework of Theorem 5.1 let $S_{1}$ be single-valued and Lipschitz continuous near $\bar{x}$. Further suppose that multifunction (25) is metrically subregular at $\left(\bar{x}, S_{1}(\bar{x}), \bar{u}, 0,0\right)$ in directions $(h, k, l)$ with $k \in D S_{1}(\bar{x})(h)$ such that $(k, l) \in$ $T_{g p h S_{2}}\left(S_{1}(\bar{x}), \bar{u}\right)$. Then inclusion (26) attains the form

$$
D^{*} S((\bar{x}, \bar{u}) ;(h, l)) \subset \bigcup_{\substack{k \in\left\{\xi \in D S_{1}(\bar{x})(h) \mid \\ l \in D S_{2}\left(S_{1}(\bar{x}), \bar{u}\right)(\xi)\right\}}} D^{*} S_{1}(\bar{x} ;(h, k)) \circ D^{*} S_{2}\left(\left(S_{1}(\bar{x}), \bar{u}\right) ;(k, l)\right) .
$$

If, moreover, $S_{1}$ is directionally differentiable at $\bar{x}$, then

$$
D^{*} S((\bar{x}, \bar{u}) ;(h, l)) \subset D^{*} S_{1}(\bar{x} ;(h, k)) \circ D^{*} S_{2}\left(\left(S_{1}(\bar{x}), \bar{u}\right) ;(k, l)\right),
$$

where $k=S_{1}^{\prime}(\bar{x} ; h)$.

Note that the single-valuedness and the Lipschitz continuity of $S_{1}$ are carried over to the intermediate mapping $\Xi$, yielding the fulfillment of assumption (a) as well as the reduction in the estimate. It is not difficult to verify that the properties of $S_{1}$ enable us to simplify the multifunction (25) by replacing its first row by $S_{1}(x)-w$. We make use of this fact in the formulation of Theorem 5.2.

Let us illustrate the above results via a simple example.

Example 5.1 Consider the composition

$$
S(x):=\left(N_{\mathbb{R}_{-}^{s}} \circ \varphi\right)(x),
$$

where $\varphi: \mathbb{R}^{n} \rightarrow \mathbb{R}^{s}$ is Lipschitz continuous near $\bar{x}$ and directionally differentiable at $\bar{x}$. Let $\bar{u} \in S(\bar{x})$ and directions $(h, l) \in \mathbb{R}^{n} \times \mathbb{R}^{s}$ be given and assume that the multifunction

$$
F(x, w, u):=\left[\begin{array}{l}
\varphi(x)-w \\
\operatorname{gph} N_{\mathbb{R}_{-}^{s}}-(w, u)
\end{array}\right]
$$

is metrically subregular at $(\bar{x}, \varphi(\bar{x}), \bar{u}, 0,0)$ in direction $\left(h, \varphi^{\prime}(\bar{x} ; h), l\right)$. Then, by virtue of Corollary 5.1 one has

$D^{*} S((\bar{x}, \bar{u}) ;(h, l)) \subset\left\{D^{*} \varphi\left(\bar{x} ;\left(h, \varphi^{\prime}(\bar{x} ; h)\right)\right)\left(y^{*}\right) \mid y^{*} \in D^{*} N_{\mathbb{R}_{-}^{s}}\left((\varphi(\bar{x}), \bar{u}) ;\left(\varphi^{\prime}(\bar{x} ; h), l\right)\right)\right\}$.

As an illustration of this formula put $n=s=1$,

$$
\varphi(x)=\left\{\begin{aligned}
-0.5 x & \text { for } x \leq 0 \\
-x & \text { otherwise }
\end{aligned}\right.
$$

$\bar{x}=\bar{u}=0, h=1, l=0$. Clearly, $\varphi^{\prime}(\bar{x} ; h)=-1$ and the qualification condition is fulfilled because of the polyhedrality of the respective mapping (36). After an easy computation we now obtain that for any $b \in \mathbb{R}$

$$
D^{*} N_{\mathbb{R}_{-}^{s}}((0,0) ;(-1,0))(b) \subset\{0\} \text { and } D^{*} \varphi(0 ;(1,-1))(0)=\{0\} .
$$

Hence, $D^{*} S((0,0) ;(1,0))(b) \subset\{0\}$ and we are done.

Corollary 5.2 In the framework of Theorem 5.1 let $S_{2}$ be single-valued and Lipschitz continuous near every $w \in S_{1}(\bar{x})$. Further, let assumption (a) be fulfilled. Then inclusion (26) with evident simplifications holds true. 
The assumptions imposed on $S_{2}$ justify assumption (b), since for a single-valued mapping Lipschitz continuity and the Aubin property coincide.

Corollaries 5.1 and 5.2 represent our main tool in the proof of the next statement. We consider there mappings $S_{i}: \mathbb{R}^{n} \rightrightarrows \mathbb{R}^{m}, i=1,2, \ldots, p$, and associate with them the multifunction $\Xi: \mathbb{R}^{n} \times \mathbb{R}^{m} \rightrightarrows\left(\mathbb{R}^{m}\right)^{p}$ defined by

$$
\Xi(x, u)=\left\{w=\left(w_{1}, w_{2}, \ldots, w_{p}\right) \in\left(\mathbb{R}^{m}\right)^{p} \mid w_{i} \in S_{i}(x), \sum_{i=1}^{p} w_{i}=u\right\} .
$$

Theorem 5.2 (Directional coderivative sum rule) Suppose $S=S_{1}+S_{2}+\ldots+S_{p}$ for osc mappings $S_{i}: \mathbb{R}^{n} \rightrightarrows \mathbb{R}^{m}$. Let $\bar{x} \in \operatorname{dom} S, \bar{u} \in S(\bar{x})$ and $(h, l) \in \mathbb{R}^{n} \times \mathbb{R}^{m}$ be a given pair of directions. Further assume that

(a) there is a directional neighborhood $\mathcal{U}$ of $(h, l)$ such that $\Xi((\bar{x}, \bar{u})+\mathcal{U})$ is bounded;

(b) the mapping

$$
F(x, v, w)=\left[\begin{array}{l}
(x, \ldots, x)-\left(v_{1}, \ldots, v_{p}\right) \\
g p h S_{1}-\left(v_{1}, w_{1}\right) \\
\vdots \\
g p h S_{p}-\left(v_{p}, w_{p}\right)
\end{array}\right]
$$

is metrically subregular at $(\bar{x},(\bar{x}, \ldots, \bar{x}), \tilde{w}, 0,0, \ldots, 0)$ for all vectors $\tilde{w} \in \Xi(\bar{x}, \bar{u})$ in all directions $\left(h,(h, \ldots, h), k_{1}, \ldots, k_{p}\right)$ such that

$$
k_{i} \in D S_{i}(\bar{x})(h), i=1, \ldots, p, k_{1}+\ldots+k_{p}=l,
$$

and in all directions $\left(0,(0, \ldots, 0), k_{1}, \ldots, k_{p}\right)$ such that

$$
k_{i} \in D S_{i}(\bar{x})(0), i=1, \ldots, p, k_{1}+\ldots+k_{p}=0 \text { and } k:=\left(k_{1}, \ldots, k_{p}\right) \in \mathbb{S} .
$$

Then one has

$$
\begin{aligned}
D^{*} S((\bar{x}, \bar{u}) ;(h, l)) \subset \bigcup_{\tilde{w} \in \Xi(\bar{x}, \bar{u})} & \left(\bigcup_{\substack{k_{i} \in D S_{i}\left(\bar{x}, \tilde{w}_{i}\right)(h), \sum k_{i}=l}} D^{*} S_{1}\left(\left(\bar{x}, \tilde{w}_{1}\right) ;\left(h, k_{1}\right)\right)+\ldots+D^{*} S_{p}\left(\left(\bar{x}, \tilde{w}_{p}\right) ;\left(h, k_{p}\right)\right)\right. \\
& \left.\cup \bigcup_{\substack{k_{i} \in D S_{i}\left(\bar{x}, \tilde{w}_{i}\right)(0), \sum k_{i}=0, k \in \mathbb{S}}} D^{*} S_{1}\left(\left(\bar{x}, \tilde{w}_{1}\right) ;\left(0, k_{1}\right)\right)+\ldots+D^{*} S_{p}\left(\left(\bar{x}, \tilde{w}_{p}\right) ;\left(0, k_{p}\right)\right)\right) .
\end{aligned}
$$

Proof Following [22, Theorem 10.41], we observe that $S=F_{2} \circ S_{o} \circ F_{1}$ for $S_{o}$ : $\left(x_{1}, x_{2}, \ldots, x_{p}\right) \mapsto S_{1}\left(x_{1}\right) \times \ldots \times S_{p}\left(x_{p}\right), F_{1}: x \mapsto(x, \ldots, x)(p$ copies $)$ and $F_{2}$ : $\left(u_{1}, \ldots, u_{p}\right) \mapsto u_{1}+\ldots+u_{p}$. So, it suffices to apply first Corollary 5.2 to the composition $F_{2} \circ G$ for $G=S_{o} \circ F_{1}$ (which is possible under condition (a)) and, thereafter, Corollary 5.1 to compute the directional limiting coderivative of $G$. This can be done under assumption (b) and leads directly to formula (37).

Similarly as in Theorem 5.1, the union over $\left\{k \in \mathbb{S} \mid k_{1}+\ldots+k_{p}=0, k_{i} \in\right.$ $\left.D S_{i}\left(\bar{x}, \tilde{w}_{i}\right)(0)\right\}$ vanishes provided we strengthen assumption (a) by asking that the intermediate mapping $\Xi$ is inner calm at $(\bar{x}, \bar{u}, \tilde{w})$ (w.r.t. gph $S$ ) in direction $(h, l)$.

Condition (b) can be ensured by the assumptions (i),(ii) below, which follow from implications (32), (33) applied to the composition $S_{o} \circ F_{1}$. They attain the form: 
(i) For all $\tilde{w} \in \Xi(\bar{x}, \bar{u})$ and all directions $k_{1}, \ldots, k_{p}$ such that $k_{i} \in D S_{i}\left(\bar{x}, \tilde{w}_{i}\right)(h), i=$ $1, \ldots, p, k_{1}+\ldots+k_{p}=l$, one has

$$
v_{i}^{*} \in D^{*} S_{i}\left(\left(\bar{x}, \tilde{w}_{i}\right) ;\left(h, k_{i}\right)\right)(0), \sum_{i=1}^{p} v_{i}^{*}=0 \quad \Longrightarrow \quad v_{i}^{*}=0 \text { for } i=1, \ldots, p .
$$

(ii) For all $\tilde{w} \in \Xi(\bar{x}, \bar{u})$ and all directions $k_{1}, \ldots, k_{p}$ such that $k_{i} \in D S_{i}\left(\bar{x}, \tilde{w}_{i}\right)(0), i=$ $1, \ldots, p, k_{1}+\ldots+k_{p}=0, k \in \mathbb{S}$, one has

$$
v_{i}^{*} \in D^{*} S_{i}\left(\left(\bar{x}, \tilde{w}_{i}\right) ;\left(0, k_{i}\right)\right)(0), \sum_{i=1}^{p} v_{i}^{*}=0 \quad \Longrightarrow \quad v_{i}^{*}=0 \text { for } i=1, \ldots, p .
$$

These conditions represent a directional version of [22, Theorem 10.41, condition (b)].

Corollary 5.3 In the setting of Theorem 5.2 assume that $p=2$ and $S_{1}$ is single-valued and Lipschitz continuous near $\bar{x}$ and directionally differentiable at $\bar{x}$. Then all assumptions of Theorem 5.2 are fulfilled and

$$
D^{*} S((\bar{x}, \bar{u}) ;(h, l)) \subset D^{*} S_{1}(\bar{x} ;(h, k))+D^{*} S_{2}\left(\left(\bar{x}, \bar{u}-S_{1}(\bar{x})\right) ;(h, l-k)\right),
$$

where $k=S_{1}(\bar{x} ; h)$.

Proof First we observe that $\Xi(\bar{x}, \bar{u})=\left\{\left(S_{1}(\bar{x}), \bar{u}-S_{1}(\bar{x})\right)\right\}$ and all assumptions of Theorem 5.2 are fulfilled thanks to the assumed properties of $S_{1}$. Formula (38) follows directly from inclusion (37).

Note that inclusion (38) becomes equality provided $S_{1}$ is continuously differentiable near $\bar{x}$, cf. [11, formula(2.4)].

The next statement is a directional version of the useful scalarization formula for singlevalued Lipschitz continuous mappings, cf. [22, Proposition 9.24] and [20, Theorem 3.28].

Proposition 5.1 Consider a single-valued continuous mapping $\varphi: \mathbb{R}^{n} \rightarrow \mathbb{R}^{m}$ which is also Lipschitz continuous near $\bar{x}$ in direction $u$. Then for any $y^{*}, v \in \mathbb{R}^{m}$ one has

$$
D^{*} \varphi(\bar{x} ;(u, v))\left(y^{*}\right)=\partial\left\langle y^{*}, \varphi\right\rangle\left(\bar{x} ;\left(u,\left\langle y^{*}, v\right\rangle\right)\right) .
$$

Proof Let $x^{*} \in D^{*} \varphi(\bar{x} ;(u, v))\left(y^{*}\right)$, i.e., $\left(x^{*},-y^{*}\right) \in N_{\operatorname{gph} \varphi}((\bar{x}, \varphi(\bar{x})) ;(u, v))$ and consider sequences $\left(t_{k}\right) \downarrow 0,\left(u_{k}, v_{k}\right) \rightarrow(u, v),\left(x_{k}^{*},-y_{k}^{*}\right) \rightarrow\left(x^{*},-y^{*}\right)$ with $\left(x_{k}^{*},-y_{k}^{*}\right) \in$ $\hat{N}_{\mathrm{gph} \varphi}\left((\bar{x}, \varphi(\bar{x}))+t_{k}\left(u_{k}, v_{k}\right)\right)$. Thus, we have $v_{k}=\left(\varphi\left(\bar{x}+t_{k} u_{k}\right)-\varphi(\bar{x})\right) / t_{k}$ and, taking into account that $\varphi$ is Lipschitz continuous near $\bar{x}+t_{k} u_{k}$ and applying [22, Proposition 9.24(b)], we obtain

$$
x_{k}^{*} \in \hat{D}^{*} \varphi\left(\bar{x}+t_{k} u_{k}\right)\left(y_{k}^{*}\right)=\hat{\partial}\left\langle y_{k}^{*}, \varphi\right\rangle\left(\bar{x}+t_{k} u_{k}\right)=\hat{\partial}\left[\left\langle y^{*}, \varphi\right\rangle+\left\langle y_{k}^{*}-y^{*}, \varphi\right\rangle\right]\left(\bar{x}+t_{k} u_{k}\right) .
$$

The fuzzy sum rule [20, Theorem 2.33] yields the existence of $x_{1}, x_{2}$ with $\| x_{i}-(\bar{x}+$ $\left.t_{k} u_{k}\right) \| \leq t_{k}^{2}$ for $i=1,2$ such that

$$
x_{k}^{*} \in \hat{\partial}\left\langle y^{*}, \varphi\right\rangle\left(x_{1}\right)+\hat{\partial}\left\langle y_{k}^{*}-y^{*}, \varphi\right\rangle\left(x_{2}\right) \subset \hat{\partial}\left\langle y^{*}, \varphi\right\rangle\left(x_{1}\right)+\left(\left\|y_{k}^{*}-y^{*}\right\| \kappa+t_{k}^{2}\right) \mathbb{B},
$$

where $\kappa$ denotes the Lipschitz constant. Consequently, there exists $\xi_{k}^{*} \in \hat{\partial}\left\langle y^{*}, \varphi\right\rangle\left(x_{1}\right)$ with $\xi_{k}^{*} \rightarrow x^{*}$ and similarly as in the proof of Theorem 3.1 one can easily show that $\tilde{u}_{k}:=$ $\left(x_{1}-\bar{x}\right) / t_{k} \rightarrow u$ and Lipschitz continuity of $\varphi$ then implies also $\left(\varphi\left(x_{1}\right)-\varphi(\bar{x})\right) / t_{k} \rightarrow v$. Thus, we conclude

$$
\left(\xi_{k}^{*},-1\right) \in \hat{N}_{\text {epi }\left\langle y^{*}, \varphi\right\rangle}\left(\bar{x}+t_{k} \tilde{u}_{k},\left\langle y^{*}, \varphi\right\rangle(\bar{x})+t_{k}\left\langle y^{*},\left(\varphi\left(\bar{x}+t_{k} \tilde{u}_{k}\right)-\varphi(\bar{x})\right) / t_{k}\right\rangle\right),
$$

showing $x^{*} \in \partial\left\langle y^{*}, \varphi\right\rangle\left(\bar{x} ;\left(u,\left\langle y^{*}, v\right\rangle\right)\right)$. 
The reverse inclusion follows easily from Theorem 4.1 since $\left\langle y^{*}, \varphi\right\rangle=g \circ \varphi$ for linear function $g=\left\langle y^{*}, \cdot\right\rangle$. Note that $\partial^{\infty} g(\varphi(\bar{x}))=\{0\}$ implies metric subregularity of $F(x, \alpha)=$ epi $g-(\varphi(x), \alpha)$.

An equivalent formulation of the following useful result was also proven in [19, Corollary 5.9].

Corollary 5.4 For an lsc function $f: \mathbb{R}^{n} \rightarrow \overline{\mathbb{R}}$ that is also Lipschitz continuous near $\bar{x}$ in direction $h$ one has $\partial^{\infty} f(\bar{x} ;(h, v)) \subset\{0\}$ for all $v \in \mathbb{R}$.

Proof Indeed, $\partial^{\infty} f(\bar{x} ;(h, v)) \subset D^{*} f(\bar{x} ;(h, v))(0)=\partial\langle 0, f\rangle(\bar{x} ;(h,\langle 0, v\rangle))=\{0\}$.

\section{Applications}

In this section we apply some of the above presented calculus rules to several problems of variational analysis, where directional notions can be advantageously utilized.

\subsection{First-order sufficient conditions for directional metric regularity and subregularity of feasibility mappings}

Consider a mapping of the form $F(x)=\Omega-\varphi(x)$ which arises in qualification conditions throughout the whole paper. The next result (announced already in Section 2) extends the results from [10, Theorem 1, Corollary 1], where $\varphi$ is assumed to be continuously differentiable.

Theorem 6.1 Let the multifunction $F: \mathbb{R}^{n} \rightrightarrows \mathbb{R}^{m}$ be given by $F(x)=\Omega-\varphi(x)$, where $\varphi: \mathbb{R}^{n} \rightarrow \mathbb{R}^{m}$ is continuous and $\Omega \subset \mathbb{R}^{m}$ is a closed set. Further let $(\bar{x}, 0) \in$ gph $F$, $(u, v) \in \mathbb{R}^{n} \times \mathbb{R}^{m}$ be given and assume that $\varphi$ is calm at $\bar{x}$ in direction $u$. Then

1. $F$ is metrically subregular at $(\bar{x}, 0)$ in direction $u$ provided for all $w \in D \varphi(\bar{x})(u) \cap$ $T_{\Omega}(\varphi(\bar{x}))$ one has the implication

$$
0 \in D^{*} \varphi(\bar{x} ;(u, w))(\lambda), \lambda \in N_{\Omega}(\varphi(\bar{x}) ; w) \Longrightarrow \lambda=0 .
$$

2. $F$ is metrically regular at $(\bar{x}, 0)$ in direction $(u, v)(c f .[6$, Definition 1]) provided for all $w \in D \varphi(\bar{x})(u)$ with $w+v \in T_{\Omega}(\varphi(\bar{x}))$ one has the implication

$$
0 \in D^{*} \varphi(\bar{x} ;(u, w))(\lambda), \lambda \in N_{\Omega}(\varphi(\bar{x}) ; v+w) \Longrightarrow \lambda=0 .
$$

The proof of the second statement is based on [6, Theorem 5] which provides equivalent characterizations of directional metric regularity and in finite dimensional spaces one of them states that $F$ is metrically regular at $(\bar{x}, 0)$ in direction $(u, v)$ if and only if $0 \in D^{*} F((\bar{x}, 0) ;(u, v))(\lambda)$ implies $\lambda=0$. The first statement then follows from the fact that metric regularity of $F$ at $(\bar{x}, 0)$ in direction $(u, 0)$ implies metric subregularity of $F$ at $(\bar{x}, 0)$ in direction $u$. Thus, it suffices to show the following lemma.

Lemma 6.1 Under the settings and assumptions of the previous theorem we have

$$
D^{*} F((\bar{x}, 0) ;(u, v))(-\lambda) \subset \bigcup_{\substack{w \in\left\{\omega \in D \varphi(\bar{x})(u) \mid \\ \lambda \in N_{\Omega}(\varphi(\bar{x}) ; v+\omega)\right\}}} D^{*} \varphi(\bar{x} ;(u, w))(\lambda) .
$$


Proof The assumed calmness of $\varphi$ implies that the intermediate mapping $\Xi(x, y)=\{y+$ $\varphi(x),-\varphi(x)\}$ is inner calm at $(\bar{x}, 0, \varphi(\bar{x}),-\varphi(\bar{x}))$ in direction $(u, v)$. On the other hand, denoting by $G$ the mapping that to each $x$ assigns set $\Omega$, it is clear that $G$ has the Aubin property and we may apply the sum rule for coderivatives, Theorem 5.2. The statement of the lemma thus follows from the fact that for some $w$ we obtain

$$
\begin{aligned}
D^{*} G((\bar{x}, \varphi(\bar{x})) ;(u, v+w))(-\lambda) & =\left\{\xi \mid(\xi, \lambda) \in N_{\mathbb{R}^{n} \times \Omega}((\bar{x}, \varphi(\bar{x})) ;(u, v+w))\right\} \\
& = \begin{cases}0 & \text { if } \lambda \in N_{\Omega}(\varphi(\bar{x}) ; v+w), \\
\emptyset & \text { otherwise. }\end{cases}
\end{aligned}
$$

\subsection{Subtransversality of set systems}

Consider the collection of closed sets $C_{1}, C_{2}, \ldots, C_{l}$ from $\mathbb{R}^{n}$ and a point $\bar{x} \in C:=\bigcap_{i=1}^{l} C_{i}$. By the definition (cf., e.g., [18, Definition 1(ii)]), these sets are subtransversal at $\bar{x}$ provided there exist a neighborhood $U$ of $\bar{x}$ and a constant $L>0$ such that the metric inequality

$$
d_{C}(x) \leq L \sum_{i=1}^{l} d_{C_{i}}(x)
$$

holds for all $x \in U$. This is, on the other hand, equivalent with the calmness of the perturbation mapping

$$
S\left(p_{1}, \ldots, p_{l}\right)=\left\{x \mid p_{i}+x \in C_{i}, i=1,2, \ldots, l\right\}
$$

at $(0, \ldots, 0, \bar{x})$, cf. [16, Section 3]. A straightforward application of [11, Theorem 3.8] yields the following result.

Theorem 6.2 Assume that there do not exist nonzero vectors $u \in \mathbb{R}^{n}$ and $v^{*}=$ $\left(v_{1}^{*}, \ldots, v_{l}^{*}\right) \in \mathbb{R}^{l n}$, such that

$$
u \in \bigcap_{i=1}^{l} T_{C_{i}}(\bar{x}), \quad 0=\sum_{i=1}^{l} v_{i}^{*}, \quad v_{i}^{*} \in N_{C_{i}}(\bar{x}, u), i=1,2, \ldots, l .
$$

Then collection $\left\{C_{1}, C_{2}, \ldots, C_{l}\right\}$ is subtransversal at $\bar{x}$.

Very often the sets $C_{i}$ correspond to various constraint systems and can be described as

$$
C_{i}=\varphi_{i}^{-1}\left(Q_{i}\right)
$$

with $Q_{i} \subset \mathbb{R}^{m_{i}}$ being closed and $\varphi_{i}: \mathbb{R}^{n} \rightarrow \mathbb{R}^{m}$ being Lipschitz continuous near $\bar{x}$. As a simple consequence of Theorem 6.2 we obtain a condition ensuring the subtransversality of a collection of pre-images.

Corollary 6.1 In the setting of Theorem 6.2 assume that the sets $C_{i}$ are given via (39) where, in addition to the posed assumptions, functions $\varphi_{i}$ are directionally differentiable at $\bar{x}$. Further assume that the mappings

$$
F_{i}(x)=Q_{i}-\varphi_{i}(x), i=1,2, \ldots, l,
$$


are metrically subregular at $\bar{x}$. Finally suppose that there do not exist nonzero vectors $u \in$ $\mathbb{R}^{n}$ and $v^{*}=\left(v_{1}^{*}, \ldots, v_{l}^{*}\right) \in \mathbb{R}^{l n}$, such that

$$
\begin{aligned}
& u \in\left\{h \in \mathbb{R}^{n} \mid \varphi_{i}^{\prime}(\bar{x} ; h) \in T_{Q_{i}}(\varphi(\bar{x})), i=1,2, \ldots, l\right\}, \\
& 0=\sum_{i=1}^{l} v_{i}^{*}, \\
& v_{i}^{*} \in D^{*} \varphi_{i}\left(\bar{x} ;\left(u, \varphi_{i}^{\prime}(\bar{x} ; u)\right)\right) N_{Q_{i}}\left(\varphi_{i}(\bar{x}) ; \varphi_{i}^{\prime}(\bar{x} ; u)\right), i=1,2, \ldots, l .
\end{aligned}
$$

Then collection $\left\{C_{1}, C_{2}, \ldots, C_{l}\right\}$ is subtransversal at $\bar{x}$.

The proof follows easily from Theorem 6.2, Theorem 3.1 and the fact that the set on the right-hand side of (40) amounts exactly to $\bigcap_{i=1}^{l} T_{C_{i}}(\bar{x})$.

Example 6.1 Let $n=2, l=2$,

$$
\begin{aligned}
& C_{1}=\left\{x \times \mathbb{R}^{2} \mid-x_{1}-x_{1}^{2}+x_{2} \leq 0,-x_{1}-x_{1}^{2}-x_{2} \leq 0\right\}, \\
& C_{2}=\left\{x \times \mathbb{R}^{2} \mid\left(\begin{array}{r}
x_{2} \\
-x_{1}
\end{array}\right) \in \operatorname{gph} N_{\mathbb{R}_{+}}\right\},
\end{aligned}
$$

and $\bar{x}=(0,0)$. It is easy to verify that all assumptions of Corollary 6.1 are fulfilled and the only direction $u$ satisfying (40) is the direction $\mathbb{R}_{+}(1,0)$. Clearly, with $\varphi_{1}$ and $Q_{1}$ given in (43) one has

$$
\nabla \varphi_{1}(\bar{x})^{T} N_{Q_{1}}\left(\varphi_{1}(\bar{x}) ; \nabla \varphi_{1}(\bar{x}) u\right)=\left[\begin{array}{rr}
-1 & -1 \\
1 & -1
\end{array}\right] N_{\mathbb{R}_{-}^{2}}((0,0) ;(-1,-1))=\{(0,0)\} .
$$

Consequently, there does not exist any nonzero pair $v_{1}^{*}, v_{2}^{*}$ satisfying conditions (41), (42) and so collection $\left\{C_{1}, C_{2}\right\}$ is subtransversal at $\bar{x}$.

Note that we are not able to detect this property via the (stronger) Aubin property of $S$ because, with $\bar{p}=0$,

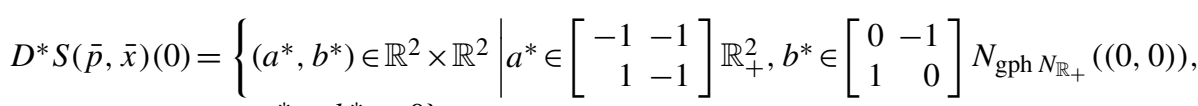

$$
\begin{aligned}
& \left.a^{*}+b^{*}=0\right\} \text {. }
\end{aligned}
$$

Thus, since the vectors $a^{*}=(-1,0)$ and $b^{*}=(1,0)$ belong to $D^{*} S(\bar{p}, \bar{x})(0)$, we conclude from the Mordukhovich criterion that $S$ does not possess the Aubin property around $(\bar{p}, \bar{x})$. $\triangle$

\subsection{Aubin property of implicitly given mappings}

By combination of [11, Theorem 4.4] with Proposition 5.1 one obtains a sufficient condition for the Aubin property for a class of implicitly defined multifunctions. Let the function $M: \mathbb{R}^{l} \times \mathbb{R}^{n} \rightarrow \mathbb{R}^{m}$ be Lipschitz continuous near the reference point $(\bar{p}, \bar{x}) \in \mathbb{R}^{l} \times \mathbb{R}^{n}$ satisfying $M(\bar{p}, \bar{x})=0$ and consider the solution mapping

$$
S(p):=\left\{x \in \mathbb{R}^{n} \mid M(p, x)=0\right\} .
$$

Theorem 6.3 Assume that $M$ is directionally differentiable at $(\bar{p}, \bar{x})$ and

(i) $\left\{u \in \mathbb{R}^{n} \mid M^{\prime}((\bar{p}, \bar{x}) ;(v, u))=0\right\} \neq \emptyset$ for allv $\in \mathbb{R}^{l}$;

(ii) $M$ is metrically subregular at $(\bar{p}, \bar{x}, 0)$; 
(iii) For every nonzero $(v, u) \in \mathbb{R}^{l} \times \mathbb{R}^{n}$ such that $M^{\prime}((\bar{p}, \bar{x}) ;(v, u))=0$ one has the implication

$$
\left[\begin{array}{l}
q^{*} \\
0
\end{array}\right] \in \partial\left\langle y^{*}, M\right\rangle((\bar{p}, \bar{x}) ;(v, u, 0)) \Rightarrow q^{*}=0 .
$$

Then $S$ has the Aubin property around $(\bar{p}, \bar{x})$.

This statement remains valid if conditions (ii), (iii) are replaced by the (stronger) implication

$$
\left[\begin{array}{l}
q^{*} \\
0
\end{array}\right] \in \partial\left\langle y^{*}, M\right\rangle((\bar{p}, \bar{x}) ;(v, u, 0)) \Rightarrow y^{*}=0 .
$$

This result can be applied to parameterized nonlinear complementarity problems (NCPs) governed by the equation

$$
0=M(p, x):=\min \{G(p, x), H(p, x)\},
$$

where functions $G, H: \mathbb{R}^{l} \times \mathbb{R}^{n} \rightarrow \mathbb{R}^{n}$ are Lipschitz continuous near $(\bar{p}, \bar{x})$, directionally differentiable at $(\bar{p}, \bar{x})$ and the "minimum" is taken componentwise. As always in the treatment of finite-dimensional NCPs we introduce for $(p, x) \in \operatorname{gph} S$ the index sets

$$
\begin{aligned}
& I_{G}(p, x):=\left\{i \in\{1,2, \ldots, n\} \mid G_{i}(p, x)=0, H_{i}(p, x)>0\right\} \\
& I_{H}(p, x):=\left\{i \in\{1,2, \ldots, n\} \mid G_{i}(p, x)>0, H_{i}(p, x)=0\right\} \\
& I_{0}(p, x):=\left\{i \in\{1,2, \ldots, n\} \mid G_{i}(p, x)=H_{i}(p, x)=0\right\},
\end{aligned}
$$

which create a partition of $\{1,2, \ldots, n\}$. To be able to apply Theorem 6.3 to $M$ given by (45) we observe that $M^{\prime}((\bar{p}, \bar{x}) ;(v, u))$ (for general directions $\left.(v, u)\right)$ amounts to the vector $b$ such that

$$
b_{i}=\left\{\begin{array}{l}
G_{i}^{\prime}((\bar{p}, \bar{x}) ;(v, u)) \text { for } i \in I_{G}(\bar{p}, \bar{x}) \cup I_{0 G}(\bar{p}, \bar{x}) \\
H_{i}^{\prime}((\bar{p}, \bar{x}) ;(v, u)) \text { for } i \in I_{H}(\bar{p}, \bar{x}) \cup I_{0 H}(\bar{p}, \bar{x}) \\
G_{i}^{\prime}((\bar{p}, \bar{x}) ;(v, u))=H_{i}^{\prime}((\bar{p}, \bar{x}) ;(v, u)) \text { otherwise }
\end{array}\right.
$$

where $I_{0 G}(\bar{p}, \bar{x}):=\left\{j \quad \in \quad I_{0}(\bar{p}, \bar{x}) \mid G_{j}^{\prime}((\bar{p}, \bar{x}) ;(v, u))<H_{j}^{\prime}((\bar{p}, \bar{x}) ;(v, u))\right\}$ and $I_{0 H}(\bar{p}, \bar{x}):=\left\{j \in I_{0}(\bar{p}, \bar{x}) \mid H_{j}^{\prime}((\bar{p}, \bar{x}) ;(v, u))<G_{j}^{\prime}((\bar{p}, \bar{x}) ;(v, u))\right\}$.

Furthermore, by virtue of Propositions $4.3,4.4$ and the definition of the directional limiting subdifferential, we obtain

$$
\sum_{i \in I_{G}(\bar{p}, \bar{x}) \cup I_{0 G}(\bar{p}, \bar{x})}^{\partial\left\langle y^{*}, M\right\rangle((\bar{p}, \bar{x}) ;(v, u, 0)) \subset} \Phi_{i}\left(y_{i}^{*}\right)+\sum_{i \in I_{H}(\bar{p}, \bar{x}) \cup I_{0 H}(\bar{p}, \bar{x})} \Psi_{i}\left(y_{i}^{*}\right)+\sum_{i \in I_{0}(\bar{p}, \bar{x}) \backslash\left(I_{0 G}(\bar{p}, \bar{x}) \cup I_{0 H}(\bar{p}, \bar{x})\right)} \Theta_{i}\left(y_{i}^{*}\right),
$$

where the multifunctions $\Phi_{i}, \Psi_{i}, \Theta_{i}, i=1,2, \ldots, n$, map $\mathbb{R}$ to (subsets of) $\mathbb{R}^{n}$ and, for $a \in \mathbb{R}$, are defined by

$$
\begin{aligned}
& \Phi_{i}(a)=\left\{\begin{array}{l}
a \partial G_{i}((\bar{p}, \bar{x}) ;(v, u, 0)) \text { if } a>0 \\
|a| \partial\left(-G_{i}\right)((\bar{p}, \bar{x}) ;(v, u, 0)) \text { if } a<0 \\
0 \text { otherwise }
\end{array}\right. \\
& \Psi_{i}(a)=\left\{\begin{array}{l}
a \partial H_{i}((\bar{p}, \bar{x}) ;(v, u, 0)) \text { if } a>0 \\
|a| \partial\left(-H_{i}\right)((\bar{p}, \bar{x}) ;(v, u, 0)) \text { if } a<0 \\
0 \text { otherwise }
\end{array}\right. \\
& \Theta_{i}(a)=\left\langle\begin{array}{l}
a\left[\partial G_{i}((\bar{p}, \bar{x}) ;(v, u, 0)) \cup \partial H_{i}((\bar{p}, \bar{x}) ;(v, u, 0))\right] \text { if } a>0 \\
|a| \text { conv }\left[\partial\left(-G_{i}\right)((\bar{p}, \bar{x}) ;(v, u, 0)), \partial\left(-H_{i}\right)((\bar{p}, \bar{x}) ;(v, u, 0))\right] \text { if } a<0 \\
0 \text { otherwise. }
\end{array}\right.
\end{aligned}
$$

The usage of the above formulas is illustrated by the following nonsmooth NCP. 
Example 6.2 Consider the NCP governed by equations (45) with $l=n=1, G(p, x)=$ $x-x^{2}, H(p, x)=|p|-x-x^{2}$ and put $(\bar{p}, \bar{x})=(0,0)$. One has

$$
M^{\prime}((\bar{p}, \bar{x}) ;(v, u))=\min \{u,|v|-u\}
$$

and so assumption (i) of Theorem 6.3 is fulfilled. Implication (44) has to be verified for directions $(v, u)$ satisfying either $v \neq 0, u=0$ or $v \neq 0, u=|v|$. In the first case one has $\{1\} \in I_{0 G}(\bar{p}, \bar{x})$ and so implication (44) attains the form

$$
\left[\begin{array}{l}
q^{*} \\
0
\end{array}\right]=y^{*}\left[\begin{array}{l}
0 \\
1
\end{array}\right] \Rightarrow y^{*}=0 .
$$

In the second case one has $\{1\} \in I_{0 H}(\bar{p}, \bar{x})$ and so we have to verify the conditions

$$
\begin{aligned}
& \nexists y^{*}>0:\left[\begin{array}{l}
q^{*} \\
0
\end{array}\right] \in y^{*}\left[\begin{array}{c}
{[-1,1]} \\
-1
\end{array}\right] \\
& \nexists y^{*}<0:\left[\begin{array}{l}
q^{*} \\
0
\end{array}\right] \in\left|y^{*}\right|\left[\begin{array}{c}
\{-1\} \cup\{1\} \\
-1
\end{array}\right] .
\end{aligned}
$$

Since both these conditions are fulfilled, the corresponding mapping $S$ has the Aubin property around $(\bar{p}, \bar{x})$.

Note that the standard condition ensuring the Aubin property of $S$ on the basis of the Mordukhovich criterion is violated. Indeed, by the calculus from [20, Chapter 3] one has

$$
D^{*} M(\bar{p}, \bar{x})\left(y^{*}\right)=\partial\left\langle y^{*}, M\right\rangle(\bar{p}, \bar{x}) \subset\left\{\begin{array}{l}
y^{*}\left(\left\{\left[\begin{array}{l}
0 \\
1
\end{array}\right]\right\} \cup\left\{\left[\begin{array}{c}
{[-1,1]} \\
-1
\end{array}\right]\right\}\right) \text { if } y^{*}>0 \\
\left|y^{*}\right| \operatorname{conv}\left(\left[\begin{array}{r}
0 \\
-1
\end{array}\right],\left[\begin{array}{c}
\{-1\} \cup\{1\} \\
1
\end{array}\right]\right) \text { if } y^{*}<0 .
\end{array}\right.
$$

One can easily verify that, for instance, the nonzero pair $\left(q^{*}, y^{*}\right)=(0.5,-1)$ belongs to the set on the right-hand side of (46). Since we do not dispose with a better estimate of $D^{*} M(\bar{p}, \bar{x})\left(y^{*}\right)$, the Aubin property of $S$ around $(\bar{p}, \bar{x})$ cannot be detected in this way.

\subsection{Improving the standard calculus}

It can easily be seen that all rules presented in Sections 3-5 reduce to their counterparts from the classical generalized differential calculus provided we set the considered directions to be zero. In some cases, however, the classical rules may even be improved when one employs the appropriate results from this paper. This concerns both the restrictiveness of the imposed assumptions as well as the sharpness of the resulting estimates.

As to the former case, Proposition 6.1 below improves a statement from [22, Theorem 6.43 ] by a substantial relaxation of the assumptions.

Proposition 6.1 Consider a closed set $C \subset \mathbb{R}^{n}$ and a continuous mapping $\varphi: \mathbb{R}^{n} \rightarrow \mathbb{R}^{l}$, set $Q=\varphi(C)$ and consider $\bar{y} \in Q$. Let $\Psi: \mathbb{R}^{l} \rightrightarrows \mathbb{R}^{n}$ be given by $\Psi(y):=\varphi^{-1}(y) \cap C$ and let it be inner semicontinuous at $(\bar{y}, \bar{x})$ w.r.t. $Q$ for some $\bar{x} \in \Psi(\bar{y})$. Assume further that the set-valued mapping $F: \mathbb{R}^{l+n} \rightrightarrows \mathbb{R}^{2(l+n)}$ given by $F(y, x)=\left(\operatorname{gph} \varphi^{-1}-(y, x)\right) \times$ $\left(\left(\mathbb{R}^{l} \times C\right)-(y, x)\right)$ is metrically subregular at $((\bar{y}, \bar{x}),(0, \ldots, 0))$. Then

$$
N_{Q}(\bar{y}) \subset\left\{y^{*} \mid D^{*} \varphi(\bar{x})\left(y^{*}\right) \cap N_{C}(\bar{x}) \neq \emptyset\right\} .
$$

The statement follows from Theorem 3.2 for direction $v=0$, taking also into account Remark 3.1. Note that the second term in (14) is covered by the first one and no inner calmness assumption is thus needed. 
Next we show a possible improvement of two estimates for the limiting normal cones.

Proposition 6.2 Given a closed set $Q \subset \mathbb{R}^{m}$ and a continuously differentiable function $\varphi: \mathbb{R}^{n} \rightarrow \mathbb{R}^{m}$, consider the set $C:=\varphi^{-1}(Q)$. Assume that the set-valued mapping $F:$ $\mathbb{R}^{n} \rightrightarrows \mathbb{R}^{m}$ given by $F(x)=Q-\varphi(x)$ is metrically subregular at $(\bar{x}, 0)$ in every direction $h \in T_{C}(\bar{x}) \cap \mathbb{S}$. Then

$$
N_{C}(\bar{x}) \subset \hat{N}_{C}(\bar{x}) \cup \bigcup_{h \in T_{C}(\bar{x}) \cap \mathbb{S}} \nabla \varphi(\bar{x})^{T} N_{Q}(\varphi(\bar{x}) ; \nabla \varphi(\bar{x}) h) .
$$

The proof follows easily from Lemma 2.1 and Theorem 3.1. The increased sharpness of this estimate with respect to [22, Theorem 6.14] has been used in sufficient conditions for the Aubin property of a class of solution mappings in [11, 12]. It stays also behind the application discussed in Subsection 6.3.

A sharper estimate can be obtained in this way also in the case of normal cones to unions. Consider a family of closed sets $C_{i} \subset \mathbb{R}^{n}, i=1,2, \ldots, l$, and a point $\bar{x} \in C=\cup_{i=1}^{l} C_{i}$. The standard estimate attains the form

$$
N_{C}(\bar{x}) \subset \bigcup_{i \in I(\bar{x})} N_{C_{i}}(\bar{x}),
$$

where $I(\bar{x}):=\left\{i=1, \ldots, l \mid \bar{x} \in C_{i}\right\}$. This follows, e.g., from Proposition 3.1 with $h=0$. On the basis of Lemma 2.1, however, we obtain the estimate

$$
N_{C}(\bar{x}) \subset \bigcap_{i \in I(\bar{x})} \hat{N}_{C_{i}}(\bar{x}) \cup \bigcup_{h \in T_{C}(\bar{x}) \cap \mathbb{S}} \bigcup_{i \in I(\bar{x}, h)} N_{C_{i}}(\bar{x} ; h),
$$

where $I(\bar{x}, h):=\left\{i \in I(\bar{x}) \mid h \in T_{C_{i}}(\bar{x})\right\}$. This estimate is tighter than (47), which is demonstrated in the next example.

Example 6.3 (MPCC generating set) Let $C_{1}=\mathbb{R}_{+} \times\{0\}, C_{2}=\{0\} \times \mathbb{R}_{+}$and $\bar{x}=(0,0)$. We have

$$
N_{C_{1} \cup C_{2}}(\bar{x})=\left(\mathbb{R}_{-} \times \mathbb{R}_{-}\right) \cup\left(\{0\} \times \mathbb{R}_{+}\right) \cup\left(\mathbb{R}_{+} \times\{0\}\right),
$$

$N_{C_{1}}(\bar{x})=\left(\mathbb{R}_{-} \times \mathbb{R}\right)$ and $N_{C_{2}}(\bar{x})=\left(\mathbb{R} \times \mathbb{R}_{-}\right)$. This shows that in this case the standard calculus does not provide a tight estimate of the limiting normal cone.

On the other hand, we obtain $\bigcap_{i \in I(\bar{x})} \hat{N}_{C_{i}}(\bar{x})=\mathbb{R}_{-} \times \mathbb{R}_{-}, T_{C}(\bar{x}) \cap \mathbb{S}=\left(C_{1} \cup C_{2}\right) \cap \mathbb{S}=$ $\{(1,0),(0,1)\}$ and

$$
\begin{array}{ll}
I(\bar{x},(1,0))=\{1\}, & N_{C_{1}}(\bar{x} ;(1,0))=\{0\} \times \mathbb{R}, \\
I(\bar{x},(0,1))=\{2\}, & N_{C_{2}}(\bar{x} ;(0,1))=\mathbb{R} \times\{0\},
\end{array}
$$

which yields that the estimate based on the directional calculus is in fact exact.

\section{Conclusion}

The paper contains directional variants of almost all important rules arising in generalized differential calculus of limiting normal cones, subdifferentials and coderivatives. Naturally, these new rules exhibit a number of similarities with their classical counterparts concerning both the structure of resulting formulas as well as the associated qualification conditions. On the other hand, the directional calculus has also some specific hurdles related to the computation of images or pre-images of the given directions in the considered mappings. As 
mentioned in the Introduction, they lead either to additional terms in the respective formulas or to additional qualification conditions. Expectantly, most qualification conditions have a directional character, which follows from the fact that in this development one needs a "regular" behavior of feasibility mappings only in the relevant directions. In the rules relying (partially) on Theorem 3.2 we make use of a special "inner calmness" property which arose (under a different name) also in completely different contexts. In Section 6 we collected some representative (classes of) problems, where directional limiting objects are helpful and the results of this paper enable the user to compute them.

Acknowledgments The authors would like to express their gratitude to both reviewers as well as to the editor for their helpful suggestions. The research of the first two authors was supported by the Austrian Science Fund (FWF) under grant P29190-N32. The research of the third author was supported by the Grant Agency of the Czech Republic, projects 17-04301S and 17-08182S and the Australian Research Council, project DP160100854F.

Funding Information Open access funding provided by Johannes Kepler University Linz.

Open Access This article is distributed under the terms of the Creative Commons Attribution 4.0 International License (http://creativecommons.org/licenses/by/4.0/), which permits unrestricted use, distribution, and reproduction in any medium, provided you give appropriate credit to the original author(s) and the source, provide a link to the Creative Commons license, and indicate if changes were made.

\section{References}

1. Baier, R., Farkhi, E.: The Directed Subdifferential of DC functions. AMS Contemp. Math. 514, 27-43 (2010)

2. Baier, R., Farkhi, E., Roshchina, V.: From quasidifferentiable to directed subdifferentiable functions : Exact calculus rules. J. Optim. Theory Appl. 171, 384-401 (2016)

3. Borwein, J.M., Zhu, Q.J.: A survey of subdifferential calculus with application. Nonlinear Anal. 38, 687-773 (1999)

4. Demyanov, V., Rubinov, A.: Quasidifferentiability and related topics, nonconvex optimization and its applications. Springer, Boston (2000)

5. Dontchev, A.L., Rockafellar, R.T.: Implicit Functions and Solution Mappings. Springer, Heidelberg (2009)

6. Gfrerer, H.: On directional metric regularity, subregularity and optimality conditions for nonsmooth mathematical programs. Set-Valued Var. Anal. 21, 151-176 (2013)

7. Gfrerer, H.: On directional metric subregularity and second-order optimality conditions for a class of nonsmooth mathematical programs. SIAM J. Optim. 23, 632-665 (2013)

8. Gfrerer, H.: Optimality conditions for disjunctive programs based on generalized differentiation with application to mathematical programs with equilibrium constraints. SIAM J. Optim. 24, 898-931 (2014)

9. Gfrerer, H.: On metric pseudo-(sub)regularity of multifunctions and optimality conditions for degenerated mathematical programs. Set-Valued Var. Anal. 22, 79-115 (2014)

10. Gfrerer, H., Klatte, D.: lipschitz and hölder stability of optimization problems and generalized equations. Math. Program. Ser. A 158, 35-75 (2016)

11. Gfrerer, H., Outrata, J.V.: On Lipschitzian properties of implicit multifunction. SIAM J. Optim. 26, 2160-2189 (2016)

12. Gfrerer, H., Outrata, J.V.: On the Aubin property of a class of parameterized variational systems. Math. Meth. Oper. Res. 86, 443-467 (2017)

13. Gfrerer, H., Ye, J.J.: New constraints qualifications for mathematical program with equilibrium constraints via variational analysis. SIAM J. Optim. 27, 842-865 (2017)

14. Ginchev, I., Mordukhovich, B.S.: On directionally dependent subdifferentials. C.R. Bulg. Acad. Sci. 64, 497-508 (2011)

15. Ioffe, A.D.: Variational analysis of regular mappings, springer monographs in mathematics. Springer, Cham (2017) 
16. Ioffe, A.D., Outrata, J.V.: On metric and calmness qualification conditions in subdifferential calculus. Set-Valued Anal. 16, 199-227 (2008)

17. Klatte, D., Kummer, B.: On calmness of the Argmin mapping in parametric optimization problems. J. Optim. Theory Appl. 165, 708-719 (2015)

18. Kruger, A.Y., Luke, D.R., Thao, N.H.: About subtransversality of collections of sets. Set-Valued Var. Anal. 25, 701-729 (2017)

19. Long, P., Wang, B., Yang, X.: Calculus of directional subdifferentials and coderivatives in Banach spaces. Positivity 21, 223-254 (2017)

20. Mordukhovich, B.S.: Variational Analysis and Generalized Differentiation. I: Basic theory. Springer, Berlin (2006)

21. Nesterov, Y.: Lexicographic differentiation of nonsmooth functions. Math. Program. 104, 669-700 (2005)

22. Rockafellar, R.T., Wets, R.J.-B.: Variational Analysis. Springer, Berlin (1998)

23. Thinh, V.D., Chuong, T.D.: Directionally generalized differentiation for multifunctions and applications to set-valued programming problems, Ann. Oper. Res. (2017), https://doi.org/10.1007/s10479-0172400-Z

24. Ye, J.J., Zhou, J.: Verifiable sufficient conditions for the error bound property of second-order cone complementarity problems, J. Math. Program. (2017), https://doi.org/10.1007/s10107-017-1193-9 